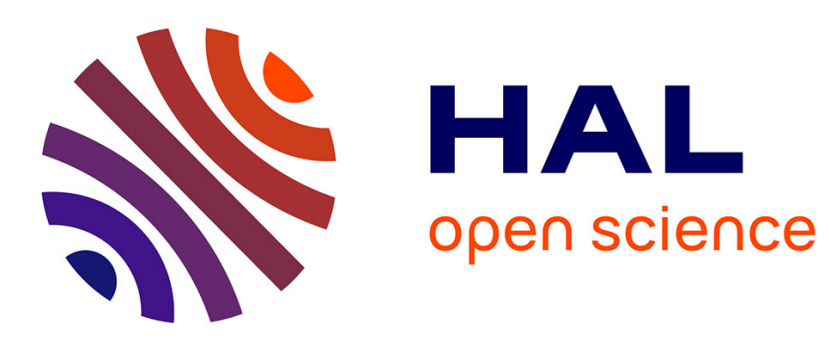

\title{
Quadratic approximation and time-varying feedback laws
}

\author{
Jean-Michel Coron, Ivonne Rivas
}

\section{To cite this version:}

Jean-Michel Coron, Ivonne Rivas. Quadratic approximation and time-varying feedback laws. SIAM Journal on Control and Optimization, 2017, 55 (6), pp.3726-3749. hal-01402747v2

\section{HAL Id: hal-01402747 \\ https://hal.science/hal-01402747v2}

Submitted on 30 Oct 2017

HAL is a multi-disciplinary open access archive for the deposit and dissemination of scientific research documents, whether they are published or not. The documents may come from teaching and research institutions in France or abroad, or from public or private research centers.
L'archive ouverte pluridisciplinaire HAL, est destinée au dépôt et à la diffusion de documents scientifiques de niveau recherche, publiés ou non, émanant des établissements d'enseignement et de recherche français ou étrangers, des laboratoires publics ou privés. 


\title{
Quadratic approximation and time-varying feedback laws
}

\author{
Jean-Michel Coron, Ivonne Rivas ${ }^{\dagger}$
}

\begin{abstract}
We present a method to construct stabilizing time-varying feedback laws for a large class of systems. We apply our technique to several classical examples which do not satisfy the necessary Brockett condition or the Coron condition for stabilization by means of continuous stationary feedback laws.
\end{abstract}

\section{Introduction}

In this paper we first study the stabilization of finite dimensional control systems of the following form

$$
\dot{x}=A x+B u \quad \text { and } \quad \dot{y}=L y+Q_{1}(x, x)+Q_{2}(x, u)+Q_{3}(u, u),
$$

where $n, m$ and $k$ are three positive integers, $A \in \mathbb{R}^{n \times n}, B \in \mathbb{R}^{n \times m}, L \in \mathbb{R}^{k \times k}$ and $Q_{1}: \mathbb{R}^{n} \times \mathbb{R}^{n} \rightarrow$ $\mathbb{R}^{k}, Q_{2}: \mathbb{R}^{n} \times \mathbb{R}^{m} \rightarrow \mathbb{R}^{k}$ and $Q_{3}: \mathbb{R}^{m} \times \mathbb{R}^{m} \rightarrow \mathbb{R}^{k}$ are bilinear maps. For the control system (1.1), the state is $\left(x^{\operatorname{tr}}, y^{\operatorname{tr}}\right)^{\operatorname{tr}} \in \mathbb{R}^{n+k}$ with $x \in \mathbb{R}^{n}$ and $y \in \mathbb{R}^{k}$ and the control is $u \in \mathbb{R}^{m}$.

There are very few physical control systems having the form given by (1.1). However, as it will be shown later on (in Theorem 4) the feedback laws, constructed to asymptotically stabilize the control system (1.1), will also asymptotically stabilize systems for which (1.1) is a "good approximation". The construction of (1.1) such that it is a "good approximation" for a given system follows from the power series expansion method introduced in [8] (see also [6, Chapter 8]) to study the local controllability of a given control system $\dot{z}=f(z, v)$ where the state is $z \in \mathbb{R}^{l}$ and the control is $v \in \mathbb{R}^{m}$. The method is the following one: let us assume that $f(0,0)=0$ and we want the study the local controllability of $\dot{z}=f(z, v)$ around $0 \in \mathbb{R}^{l}$ with small controls. We expand $z=z_{1}+z_{2}+z_{3}+\ldots, v=v_{1}+v_{2}+v_{3}+\ldots$ where $\left(z_{1}, v_{1}\right)$ is of order $1,\left(z_{2}, v_{2}\right)$ is of order $2,\left(z_{3}, v_{3}\right)$ is of order 3 and so on. Identifying the different orders in $\dot{z}=f(z, v)$ leads to

$$
\dot{z}_{1}=\frac{\partial f}{\partial z}(0,0) z_{1}+\frac{\partial f}{\partial v}(0,0) v_{1}
$$

${ }^{*}$ Université Pierre et Marie Curie-Paris 6, UMR 7598 Laboratoire Jacques-Louis Lions, 75005 Paris, France. Email: coron@ann.jussieu.fr. JMC was supported by ERC advanced grant 266907 (CPDENL) of the 7th Research Framework Programme (FP7) and by ANR Project Finite4SoS (ANR 15-CE23-0007).

${ }^{\dagger}$ Université Pierre et Marie Curie-Paris 6, UMR 7598 Laboratoire Jacques-Louis Lions, 75005 Paris, France. Email: rivas@ann.jussieu.fr. IR was supported by ERC advanced grant 266907 (CPDENL) of the 7th Research Framework Programme (FP7), by Universidad del Valle, Cali, Colombia, and by ANR Project Finite4SoS (ANR 15-CE23-0007). 


$$
\begin{aligned}
\dot{z}_{2}=\frac{\partial f}{\partial z}(0,0) z_{2}+\frac{\partial f}{\partial v}(0,0) v_{2}+\frac{1}{2} \frac{\partial^{2} f}{\partial z^{2}}(0,0)\left(z_{1}, z_{1}\right) & \\
& +\frac{\partial^{2} f}{\partial z \partial v}(0,0)\left(z_{1}, v_{1}\right)+\frac{1}{2} \frac{\partial^{2} f}{\partial v^{2}}(0,0)\left(v_{1}, v_{1}\right),
\end{aligned}
$$

and so on. The control system $(1.2)$ is a linear control systems where the state is $z_{1} \in \mathbb{R}^{l}$ and the control is $v_{1} \in \mathbb{R}^{m}$. We decompose this linear system into its controllable part and its uncontrollable part. Let $n \in\{0,1, \ldots l\}$ be the dimension of the linear controllable part. We assume that $n \in$ $\{1, \ldots l-1\}$ and let $k:=l-n \in\{1, \ldots l-1\}$. Performing, if necessary, a linear change of variables, we may assume that the controllable part is the vector space

$$
H:=\left\{z:=\left(x^{\operatorname{tr}}, y^{\operatorname{tr}}\right)^{\operatorname{tr}} \in \mathbb{R}^{l} \text { with } x \in \mathbb{R}^{n}, y=0 \in \mathbb{R}^{l-n}\right\} .
$$

Then there exists $A \in \mathbb{R}^{n \times n}, M \in \mathbb{R}^{n \times l}, L \in \mathbb{R}^{k \times k}$ and $B \in \mathbb{R}^{n \times m}$ such that

$$
\frac{\partial f}{\partial z}(0,0)=\left(\begin{array}{cc}
A & M \\
0 & L
\end{array}\right), \frac{\partial f}{\partial v}(0,0)=\left(\begin{array}{c}
B \\
0
\end{array}\right)
$$

and such that the linear control system $\dot{x}=A x+B u$, where the state is $x \in \mathbb{R}^{n}$ and the control is $u \in \mathbb{R}^{m}$, is controllable. Let us assume that $z_{1}(0) \in H$. Then $z_{1}(t)$ is in $H$ for every time $t$. We write $z_{1}=(x, 0)$ and $u=v_{1}$. From 1.2 we get

$$
\dot{x}=A x+B u .
$$

We take $v_{2}=0$. Let $y \in \mathbb{R}^{l}$ be the last $l$ components of $z_{2}: z_{2}=\left(\xi^{\operatorname{tr}}, y^{\operatorname{tr}}\right)^{\operatorname{tr}}$ for some $\xi \in \mathbb{R}^{n}$. Then (1.3) leads to

$$
\dot{y}=L y+Q_{1}(x, x)+Q_{2}(x, u)+Q_{3}(u, u),
$$

where $Q_{1}: \mathbb{R}^{n} \times \mathbb{R}^{n} \rightarrow \mathbb{R}^{k}, Q_{2}: \mathbb{R}^{n} \times \mathbb{R}^{m} \rightarrow \mathbb{R}^{k}$ and $Q_{3}: \mathbb{R}^{m} \times \mathbb{R}^{m} \rightarrow \mathbb{R}^{k}$ are bilinear maps. Note that (1.5) and (1.6) are just our initial control system (1.1). The key points of the power series expansion method is that, if (1.1) is controllable in time $T$, then $\dot{z}=f(z, v)$ is locally controllable in time $T$ and, moreover, it provides a method to check if $(1.1)$ is controllable in time $T$. This method was used in [8] to prove a controllability result on a Korteweg-de Vries equation. (In fact, in the case studied in [8], an expansion to the order 2 was not sufficient; an expansion to the order 3 was necessary.) Here we use this power series expansion in order to asymptotically stabilize $\dot{z}=f(z, v)$. Roughly, speaking under the assumptions that $0 \in \mathbb{R}^{k}$ is stable (but not necessarily asymptotically stable) for $\dot{y}=L y$, that (1.5) is controllable and that $(1.1)$ is controllable, we provide a method to construct time-varying periodic feedback laws $(t, z) \in \mathbb{R} \times \mathbb{R}^{l} \mapsto v(t, z) \in \mathbb{R}^{m}$ leading to a local exponential stability of the closed-loop system $\dot{z}=f(z, v(t, z))$ for the weighted "norm" $|x|^{2}+|y|$. We illustrate this method on some classical control systems. Note that this method can also be used in the framework of partial differential equations: See [10] for a Korteweg de Vries equation. Let us point out that the linearized control system of (1.1) around the trajectory $(\bar{x}, \bar{y}, \bar{u}):=(0,0,0)$ is

$$
\dot{x}=A x+B u \quad \text { and } \quad \dot{y}=L y,
$$

a linear control system which is never controllable. We assume the existence of $T>0$ such that the following three properties $\left(\mathcal{P}_{1}\right),\left(\mathcal{P}_{2}\right)$, and $\left(\mathcal{P}_{3}\right)$ hold

$\left(\mathcal{P}_{1}\right)$ There exists

$$
\rho_{1} \in(0,1)
$$

such that

$$
(\dot{x}=A x) \Rightarrow\left(|x(T)|^{2} \leqslant \rho_{1}|x(0)|^{2}\right) .
$$


Note that Property $\left(\mathcal{P}_{1}\right)$ implies that $0 \in \mathbb{R}^{n}$ is asymptotically stable for $\dot{x}=A x$. Conversely, if $0 \in \mathbb{R}^{n}$ is asymptotically stable for $\dot{x}=A x$, then $\left(\mathcal{P}_{1}\right)$ holds if $T>0$ is large enough (and it holds for every $T>0$ if one is allowed to perform a suitable linear invertible transformation on $x$ ). In particular if $\dot{x}=A x+B u$ is controllable, this property always holds if $T>0$ is large enough provided one replaces $A$ by $A+B K$ with a suitable $K \in \mathbb{R}^{m \times n}$. In all the applications presented below $\dot{x}=A x+B u$ is controllable.

$$
\left|e^{T L} y\right| \leq|y|, \forall y \in \mathbb{R}^{k}
$$

Note that Property $\left(\mathcal{P}_{2}\right)$ implies that $0 \in \mathbb{R}^{k}$ is stable for $\dot{y}=L y$. Conversely, if $0 \in \mathbb{R}^{k}$ is stable for $\dot{y}=L y$, performing if necessary a linear invertible transformation on $y$, (1.9) holds for every $T>0$. Let us emphasize that our results are interesting only if $0 \in \mathbb{R}^{k}$ is not asymptotically stable for $\dot{y}=L y$. Indeed if $0 \in \mathbb{R}^{k}$ is asymptotically stable for $\dot{y}=L y$ and if Property $\left(\mathcal{P}_{1}\right)$ also holds, then $0 \in \mathbb{R}^{n+k}$ is already globally asymptotically stable for (1.1) with the feedback law $u=0$ and $0 \in \mathbb{R}^{n+k}$ is already locally asymptotically stable for 1.19 with the feedback law $u_{\varepsilon}=0$ provided that 1.20 and $\left(1.21\right.$ hold. In all the applications given below, $0 \in \mathbb{R}^{k}$ is not asymptotically stable for $\dot{y}=L y$.

$\left(\mathcal{P}_{3}\right)$ There exist $\delta>0, C_{0}>0$ and a measurable function $v:[0, T] \times \mathbb{S}^{k-1} \rightarrow \mathbb{R}^{m}$ such that

$$
\begin{gathered}
|v(t, b)| \leqslant C_{0}, \forall t \in[0, T], \forall b \in \mathbb{S}^{k-1}, \\
\left|v(t, b)-v\left(t, b^{\prime}\right)\right| \leqslant C_{0}\left|b-b^{\prime}\right|, \forall t \in[0, T], \forall b \in \mathbb{S}^{k-1}, \forall b^{\prime} \in \mathbb{S}^{k-1}, \\
\left(\dot{\tilde{x}}=A \tilde{x}+B v(t, b), \dot{\tilde{y}}=L \tilde{y}+Q_{1}(\tilde{x}, \tilde{x})+Q_{2}(\tilde{x}, v(t, b))+Q_{3}(v(t, b), v(t, b)),\right. \\
\tilde{x}(0)=0, \tilde{y}(0)=0) \Rightarrow\left(\tilde{x}(T)=0, \tilde{y}(T) \cdot e^{T L} b \leqslant-2 \delta\right), \forall b \in \mathbb{S}^{k-1} .
\end{gathered}
$$

In $\left(\mathcal{P}_{3}\right)$ and in the following, $\mathbb{S}^{k-1}$ denotes the unit sphere of $\mathbb{R}^{k}: \mathbb{S}^{k-1}:=\left\{b \in \mathbb{R}^{k} ;|b|=1\right\}$. For $\varepsilon>0$, let us consider the following periodic time-varying feedback law $u_{\varepsilon}: \mathbb{R} \times \mathbb{R}^{k} \rightarrow \mathbb{R}^{m}$

$$
\begin{gathered}
u_{\varepsilon}(t, y):= \begin{cases}\varepsilon \sqrt{\left|e^{-t L} y\right|} v\left(t, \frac{e^{-t L} y}{\left|e^{-t L} y\right|}\right), & \forall t \in[0, T), \forall y \in \mathbb{R}^{k} \backslash\{0\}, \\
0, & \forall t \in[0, T), y=0 \in \mathbb{R}^{k} .\end{cases} \\
u_{\varepsilon}(t+T, y)=u_{\varepsilon}(t, y), \forall t \in \mathbb{R}, \forall y \in \mathbb{R}^{k} .
\end{gathered}
$$

In order to motivate Property $\left(\mathcal{P}_{3}\right)$, let us mention that the more popular condition of controllability implies this property. More precisely, let us first define the (classical) notion of controllability we are considering.

Definition 1 Let $\tau>0$. The control system (1.1) is locally controllable in time $\tau>0$ if there exists $\eta>0$ such that, for every $\left(x_{0}, y_{0}\right) \in \mathbb{R}^{n} \times \mathbb{R}^{k}$ such that $\left|x_{0}\right|+\left|y_{0}\right|<\eta$, there exists $u \in L^{\infty}\left([0, \tau] ; \mathbb{R}^{m}\right)$ such that

$$
\begin{array}{r}
\left(\dot{x}=A x+B u, \dot{y}=L y+Q_{1}(x, x)+Q_{2}(x, u)+Q_{3}(u, u), \quad x(0)=x_{0}, y(0)=y_{0}\right) \Rightarrow \\
(x(\tau)=0, y(\tau)=0) .
\end{array}
$$


(Let us point out that if $\left(\left(x^{\operatorname{tr}}, y^{\operatorname{tr}}\right)^{\operatorname{tr}}, u\right):[0, \tau] \rightarrow \mathbb{R}^{n+k} \times \mathbb{R}^{m}$ is a trajectory of the control system (1.1), then, for every $\lambda \in \mathbb{R},\left(\left(\lambda x^{\operatorname{tr}}, \lambda^{2} y^{\operatorname{tr}}\right)^{\operatorname{tr}}, \lambda u\right):[0, \tau] \rightarrow \mathbb{R}^{n+k} \times \mathbb{R}^{m}$ is a trajectory of the control system (1.1). Hence the local controllability in time $\tau>0$ implies in fact the global controllability in time $\tau$.) There are many explicit conditions relying on iterated Lie brackets allowing to check if the control system (1.1) is locally controllable in small time (i.e. in time $\tau$ for every $\tau>0$ ). For more details on these explicit conditions, see in particular [22] and [6, Chapter 3]. With this notion of local controllability, one has the following proposition, which is proved in Appendix A.

Proposition 2 Let us assume that there exists $\tau \in(0, T)$ such that the control system (1.1) is locally controllable in time $\tau$. Then Property $\left(\mathcal{P}_{3}\right)$ holds.

In all the applications given below, there exists indeed a $\tau \in(0, T)$ such that the control system (1.1) is locally controllable in time $\tau$.

We are interested in the asymptotic behavior of the solutions to the closed-loop system

$$
\dot{x}=A x+B u_{\varepsilon}(t, y) \quad \text { and } \quad \dot{y}=L y+Q_{1}(x, x)+Q_{2}(x, u)+Q_{3}(u, u) .
$$

Let us emphasize that the regularity of $u_{\varepsilon}$ is sufficient for the existence of solutions of the Cauchy problem associated to (1.16). Moreover, by a theorem due to Kurzweil [12], $0 \in \mathbb{R}^{n+m}$ is globally asymptotically stable for (1.16) if and only if there is a Lyapunov function (of class $C^{\infty}$ ) which is $T$-periodic with respect to time. The existence of this Lyapunov function is important since it insures some robustness with respect to (small) perturbations, which is in fact the true goal of the stabilization issue.

The following theorem, which is proved in Section 2, shows that the feedback law $u_{\varepsilon}$ defined by (1.13)-(1.14 leads to global asymptotic stability provided that $\varepsilon>0$ is small enough.

Theorem 3 Let us assume that $\left[\left(\mathcal{P}_{1}\right),\left(\mathcal{P}_{2}\right)\right.$ and $\left(\mathcal{P}_{3}\right)$ hold. Then, there exists $\varepsilon_{0}>0$ such that, for every $\varepsilon \in\left(0, \varepsilon_{0}\right]$, there exist $C>0$ and $\lambda>0$ such that, for every solution $(x, y)$ of $(1.16)$, one has

$$
|x(t)|^{2}+|y(t)| \leqslant C e^{-\lambda t}\left(|x(0)|^{2}+|y(0)|\right), \forall t \in[0,+\infty) .
$$

Our next result allows to stabilize nonlinear control systems for which the quadratic "approximation" satisfies the assumptions of Theorem 3. The control system takes now the following more general form

$$
\dot{x}=A x+B u+R_{x}(x, y, u), \dot{y}=L y+Q_{1}(x, x)+Q_{2}(x, u)+Q_{3}(u, u)+R_{y}(x, y, u),
$$

where the state is $\left(x^{\operatorname{tr}}, y^{\operatorname{tr}}\right)^{\operatorname{tr}} \in \mathbb{R}^{n+k}$, with $x \in \mathbb{R}^{n}$ and $y \in \mathbb{R}^{k}$, and the control is $u \in \mathbb{R}^{m}$. We assume that $R_{x}: \mathbb{R}^{n} \times \mathbb{R}^{k} \rightarrow \mathbb{R}^{n}$ and $R_{y}: \mathbb{R}^{n} \times \mathbb{R}^{k} \rightarrow \mathbb{R}^{k}$ are both continuous. Our next result deals with the asymptotic stability of 0 for the closed-loop system

$$
\left\{\begin{array}{l}
\dot{x}=A x+B u_{\varepsilon}(t, y)+R_{x}\left(x, y, u_{\varepsilon}(t, y)\right) \\
\dot{y}=L y+Q_{1}(x, x)+Q_{2}\left(x, u_{\varepsilon}(t, y)\right)+Q_{3}\left(u_{\varepsilon}(t, y), u_{\varepsilon}(t, y)\right)+R_{y}\left(x, y, u_{\varepsilon}(t, y)\right) .
\end{array}\right.
$$

We have the following theorem, which is proved in Section 3 .

Theorem 4 Let us assume that $\left[\left(\mathcal{P}_{1}\right),\left(\mathcal{P}_{2}\right)\right.$ and $\left(\mathcal{P}_{3}\right)$ hold. Let us also assume the existence of $\eta>0$ and $M>0$ such that, for every $(x, y, u) \in \mathbb{R}^{n} \times \mathbb{R}^{k} \times \mathbb{R}^{m}$ satisfying $|x|+|y|+|u| \leqslant 1$,

$$
\begin{gathered}
\left|R_{x}\left(\varepsilon x, \varepsilon^{2} y, \varepsilon u\right)\right| \leq M \varepsilon^{1+\eta}, \forall \varepsilon \in(0,1), \\
\left|R_{y}\left(\varepsilon x, \varepsilon^{2} y, \varepsilon u\right)\right| \leq M \varepsilon^{2+\eta}
\end{gathered}
$$


Then, there exists $\varepsilon_{0}>0$ such that, for every $\varepsilon \in\left(0, \varepsilon_{0}\right]$, there exist $C>0, \rho>0$ and $\lambda>0$ such that, for every solution $(x, y)$ of 1.19 with $|x(0)|^{2}+|y(0)| \leqslant \rho$, one has

$$
|x(t)|^{2}+|y(t)| \leqslant C e^{-\lambda t}\left(|x(0)|^{2}+|y(0)|\right), \forall t \in[0,+\infty) .
$$

Remark 5 Our method allows the construction of time-varying feedback laws. Note that, as first pointed out by Sussmann in [21] and by Brockett in [1], there are controllable systems which cannot be asymptotically stabilized by means of continuous stationary (i.e. which does not depend on time) feedback laws. To overcome this difficulty the use of time-varying feedback laws have been proposed in two pioneer works: [19] by Sontag and Sussmann for control systems with states of dimension 1, [17] by Samson for the control system studied in Section 4.3 and that we revisit with our method. General results showing that many controllable systems can be asymptotically (and even in finite time) stabilized by means of time-varying feedback laws can be found in [3, 5]. The fact that the control systems presented in Section 4 can be asymptotically stabilized by means of time-varying feedback laws follows from [3, 5]. The novelty of our approach is to allow explicit constructions of such feedback laws.

\section{Proof of Theorem 3}

Let

$$
\rho_{2} \in\left(\rho_{1},+\infty\right)
$$

Theorem 3 is a corollary of the following proposition, where assumption (1.7) is no longer required.

Proposition 6 There exists $\varepsilon_{0}>0$ and $C>0$ such that, for every $\varepsilon \in\left[0, \varepsilon_{0}\right]$ and for every solution of (1.16), one has

$$
\begin{gathered}
|x(t)|^{2}+|y(t)| \leqslant C\left(|x(s)|^{2}+|y(s)|\right), \forall s \in[0, T], \forall t \in[s, T], \\
|x(T)|^{2}+\varepsilon|y(T)| \leqslant \rho_{2}|x(0)|^{2}+\varepsilon\left(1-\varepsilon^{2} \delta\right)|y(0)| .
\end{gathered}
$$

Proof of Proposition 6. Let $\left(x^{\operatorname{tr}}, y^{\operatorname{tr}}\right)^{\operatorname{tr}}$ be a solution of $(1.16)$ on $[0, T]$. From now on, we denote by $C>0$ various constants which vary from place to place but do not depend on $\varepsilon \in(0,1)$, on $t \in[0, T]$ or on $\left(x^{\operatorname{tr}}, y^{\operatorname{tr}}\right)^{\operatorname{tr}}$ solution of (1.16). However, these constants $C$ may depend on $T, A, B$, $L$ and $v$.

From 1.10, 1.13) and (1.16), we get that

$$
\frac{d}{d t}\left(|x|^{4}+|y|^{2}\right) \leqslant C\left(|x|^{4}+|y|^{2}\right)
$$

which gives (2.2). From (1.10), 1.13), 1.16) and (2.2), we get that

$$
\left|x(t)-e^{t A} x(0)\right| \leqslant C \varepsilon(|x(0)|+\sqrt{|y(0)|}) .
$$

From 1.16) and 2.5), we have

$$
\left|y(t)-e^{t L} y(0)\right| \leqslant C\left(|x(0)|^{2}+\varepsilon^{2}|y(0)|\right) .
$$


From (1.8), 2.1), 2.5 and (2.6), there exists $\varepsilon_{1}>0$, such that for every

$$
\varepsilon \in\left[0, \varepsilon_{1}\right]
$$

one has $(2.3)$ for every solution of $(1.16)$ satisfying $|x(0)| \geqslant \varepsilon^{2 / 3} \sqrt{|y(0)|}$. From now on, we consider that (2.7) holds and that

$$
|x(0)|<\varepsilon^{2 / 3} \sqrt{|y(0)|} .
$$

Note that if $(x, y)$ is a solution of $(1.16)$, then, for every $\lambda \geqslant 0,\left(\lambda x, \lambda^{2} y\right)$ is also a solution of (1.16). Hence, using also (2.8), in order to prove (2.3), we may assume, without loss of generality, that

$$
\begin{gathered}
b:=y(0) \in \mathbb{S}^{k-1}, \\
|x(0)| \leqslant \varepsilon^{2 / 3} .
\end{gathered}
$$

Equations (2.5), 2.6), 2.9) and (2.10) lead us to

$$
|x(t)| \leqslant C \varepsilon^{2 / 3}, \quad\left|y(t)-e^{L t} b\right| \leqslant C \varepsilon^{4 / 3}, \forall t \in[0, T] .
$$

Let us define $x_{1}:[0, T] \rightarrow \mathbb{R}^{n}, x_{2}:[0, T] \rightarrow \mathbb{R}^{n}, x_{3}:[0, T] \rightarrow \mathbb{R}^{n}, r:[0, T] \rightarrow \mathbb{R}^{m}$ by

$$
\begin{gathered}
x_{1}(t):=e^{t A} x(0), \\
\dot{x}_{2}=A x_{2}+B v(t, b), x_{2}(0)=0, \\
x_{3}:=x-x_{1}-\varepsilon x_{2}, \\
r(t):=u_{\varepsilon}(t, y(t))-\varepsilon v(t, b) .
\end{gathered}
$$

From (1.8) and 2.12), one has

$$
\left|x_{1}(T)\right|^{2} \leqslant \rho_{1}|x(0)|^{2} .
$$

From 1.10, 1.12 and 2.13

$$
\begin{gathered}
\left|x_{2}(t)\right| \leqslant C, \forall t \in[0, T], \\
x_{2}(T)=0 .
\end{gathered}
$$

From (1.16), 2.12, 2.13 and 2.14), one has

$$
\dot{x}_{3}=A x_{3}+B u_{\varepsilon}(t, y(t))-\varepsilon B v(t, b), x_{3}(0)=0 .
$$

From (1.10), (1.11), 1.13), 2.11) and (2.19), one has

$$
\left|x_{3}(t)\right| \leqslant C \varepsilon^{2}, \forall t \in[0, T] .
$$

Concerning $u_{\varepsilon}$ and $r$, using (1.10), (1.11) and (2.11), one has the following estimates

$$
\left|u_{\varepsilon}(t, y(t))\right| \leqslant C \varepsilon,|r(t)| \leqslant C \varepsilon^{7 / 3}, \forall t \in[0, T] .
$$

Let us fix

$$
\rho_{3 / 2} \in\left(\rho_{1}, \rho_{2}\right)
$$


(The existence of $\rho_{3 / 2}$ follows from (2.1)). From (2.14), 2.16), 2.18), 2.20) and (2.22), one gets that

$$
|x(T)|^{2} \leqslant \rho_{3 / 2}|x(0)|^{2}+C \varepsilon^{4} .
$$

We now estimate $y$. Let $y_{1}:[0, T] \rightarrow \mathbb{R}^{k}, y_{2}:[0, T] \rightarrow \mathbb{R}^{k}$ and $y_{3}:[0, T] \rightarrow \mathbb{R}^{k}$ be defined by

$$
\begin{gathered}
\dot{y}_{1}=L y_{1}+2 Q_{1}\left(x_{1}, x\right)-Q_{1}\left(x_{1}, x_{1}\right)+Q_{2}\left(x_{1}, u\right), \quad y_{1}(0)=0, \\
\dot{y}_{2}=L y_{2}+Q_{1}\left(x_{2}, x_{2}\right)+Q_{2}\left(x_{2}, v\right)+Q_{3}(v, v), \quad y_{2}(0)=0, \\
y_{3}:=y-y_{1}-\varepsilon^{2} y_{2}-e^{t L} b .
\end{gathered}
$$

In (2.24), 2.25) and in the following, $u(t):=u_{\varepsilon}(t, y(t))$ and, with a slight abuse of notation, $v(t)$ is $v(t, b)$. Then, from 2.11, 2.12 and 2.24, one has

$$
\left|y_{1}(t)\right| \leqslant C \varepsilon^{2 / 3}|x(0)|, \forall t \in[0, T] .
$$

and (1.10), 1.12, 2.9), 2.13, 2.17) and 2.25) give us

$$
\left|y_{2}(T)\right| \leqslant C\left(1+\varepsilon^{2}\right), y_{2}(T) \cdot e^{T L} b \leqslant-2 \delta+C \varepsilon^{2} .
$$

From (1.10), 2.9 and 2.28), we have

$$
\left|e^{T L} b+\varepsilon^{2} y_{2}(T)\right| \leqslant 1-4 \delta \varepsilon^{2}+C \varepsilon^{4} .
$$

Equation 1.16, 2.14, 2.15, 2.24, 2.25 and 2.26 give us

$$
\begin{aligned}
\dot{y}_{3}= & L y_{3}+\varepsilon Q_{1}\left(x_{2}, x_{3}\right)+\varepsilon Q_{1}\left(x_{3}, x_{2}\right)+Q_{1}\left(x_{3}, x_{3}\right) \\
& +\varepsilon Q_{2}\left(x_{2}, r\right)+\varepsilon Q_{2}\left(x_{3}, v\right)+Q_{2}\left(x_{3}, r\right)+\varepsilon Q_{3}(v, r)+\varepsilon Q_{3}(r, v)+Q_{3}(r, r), \quad y_{3}(0)=0 .
\end{aligned}
$$

From 2.17, 2.20, 2.21 and 2.30, one has

$$
\left|y_{3}(T)\right| \leqslant C \varepsilon^{3} .
$$

Using (2.26), 2.27), 2.29) and (2.31), one has

$$
|y(T)| \leqslant 1-4 \delta \varepsilon^{2}+C \varepsilon^{3}+C \varepsilon^{2 / 3}|x(0)| .
$$

From (2.9), 2.22), 2.23) and 2.32), one gets that exists $\varepsilon_{0}>0$ such that, if $\varepsilon \in\left[0, \varepsilon_{0}\right]$,

$$
|x(T)|^{2}+\varepsilon|y(T)| \leqslant \rho_{2}|x(0)|^{2}+\varepsilon\left(1-2 \delta \varepsilon^{2}\right)|y(0)|,
$$

which concludes the proof of Proposition 6.

Remark 7 It follows from our proof of Theorem 3 that, in this theorem, one can take

$$
\lambda=\min \left\{-\frac{\ln \left(\rho_{2}\right)}{T},-\frac{\ln \left(1-\varepsilon_{0}^{2} \delta\right)}{T}\right\} .
$$




\section{Proof of Theorem 4}

In this section we deduce Theorem 4 from Theorem 3 and the existence of homogeneous Lyapunov function for asymptotically stable homogeneous time-varying vector fields.

Let $Z$ be a time-varying vector field of period $T$ with respect to time, i.e. $Z: \mathbb{R} \times \mathbb{R}^{n} \rightarrow \mathbb{R}^{n}$ satisfies

$$
Z(t+T, z)=Z(t, z), \forall t \in \mathbb{R}, \forall z \in \mathbb{R}^{n} .
$$

We say that $Z$ is a Carathéodory function if it satisfies the three following properties

$$
\begin{aligned}
& \forall R>0, \exists C(R)>0 \text { such that }|Z(t, z)| \leqslant C(R), \forall t \in \mathbb{R}, \forall z \in \mathbb{R}^{n} \text { such that }|z| \leqslant R, \\
& \qquad \forall z \in \mathbb{R}^{n} \text {, the function } t \in \mathbb{R} \mapsto Z(t, z) \text { is measurable, } \\
& \text { for almost every } t \in \mathbb{R} \text {, the function } z \in \mathbb{R} \mapsto Z(t, z) \text { is continuous. }
\end{aligned}
$$

Let $I$ be an interval of $\mathbb{R}$ and let $z: I \rightarrow \mathbb{R}^{n}$. As usual, we say that $z$ is a solution of $\dot{z}=Z(t, z)$ on $I$ if $z$ is absolutely continuous on every compact subinterval of $I$ and

$$
\dot{z}(t)=Z(t, z(t)) \text { for almost every } t \in I .
$$

Let us recall that Carathéodory's Theorem insures that, if $Z$ is a Carathéodory function, then, for every $t_{0} \in \mathbb{R}$ and for every $z_{0} \in \mathbb{R}^{n}$ there is an open interval $I$ containing $t_{0}$ and a solution $z: I \rightarrow \mathbb{R}^{n}$ of $\dot{z}=Z(z)$ such that $z\left(t_{0}\right)=z_{0}$.

Let $r=\left(r_{1}, \ldots, r_{n}\right)^{\operatorname{tr}} \in(0,+\infty)^{n}$ and let $Y=\left(Y_{1}, \ldots, Y_{n}\right)^{\operatorname{tr}}: \mathbb{R} \times \mathbb{R}^{n} \rightarrow \mathbb{R}^{n}$ be a time-varying vector field. One says that the time-varying vector field $Y=\left(Y_{1}, \ldots, Y_{n}\right)^{\text {tr }}$ is $r$-homogeneous of degree 0 if

$$
Y_{i}\left(t,\left(\varepsilon^{r_{1}} z_{1}, \ldots, \varepsilon^{r_{n}} z_{n}\right)^{\operatorname{tr}}\right)=\varepsilon^{r_{i}} Y_{i}\left(t, z_{1}, \ldots, z_{n}\right), \forall \varepsilon>0, \forall z=\left(z_{1}, \ldots, z_{n}\right)^{\operatorname{tr}} \in \mathbb{R}^{n}, \forall t \in \mathbb{R} .
$$

Theorem 8 ([15]) Let $T>0$. Let $Y$ be a time-varying vector field of period $T$ with respect to time. We assume that

$$
\begin{gathered}
Y \text { is a Carathéodory vector field, } \\
Y \text { is r-homogeneous. }
\end{gathered}
$$

Let

$$
\theta \in\left(\max \left\{r_{i}, 1 \leqslant i \leqslant n\right\},+\infty\right) .
$$

Then there exists a function $V \in C^{\infty}\left(\mathbb{R} \times\left(\mathbb{R}^{n} \backslash\{0\}\right) ; \mathbb{R}\right) \cap C^{1}\left(\mathbb{R} \times \mathbb{R}^{n} ; \mathbb{R}\right)$ such that

$$
\begin{gathered}
V(t, x)>V(t, 0)=0, \forall(t, x) \in \mathbb{R} \times\left(\mathbb{R}^{n} \backslash\{0\}\right), \\
V(t+T, x)=V(t, x), \forall(t, x) \in \mathbb{R} \times \mathbb{R}^{n}, \\
\lim _{|x| \rightarrow+\infty} \operatorname{Min}\{V(t, x) ; t \in \mathbb{R}\}=+\infty, \\
\frac{\partial V}{\partial t}+Y \cdot \nabla V<0 \text { in } \mathbb{R} \times\left(\mathbb{R}^{n} \backslash\{0\}\right) \times \mathbb{R}, \\
V\left(t,\left(\varepsilon^{r_{1}} x_{1}, \ldots, \varepsilon^{r_{n}} x_{n}\right)^{t r}\right)=\varepsilon^{\theta} V\left(t,\left(x_{1}, \ldots, x_{n}\right)^{t r}\right), \forall(\varepsilon, t, x) \in(0,+\infty) \times \mathbb{R} \times \mathbb{R}^{n} .
\end{gathered}
$$

As a corollary of this theorem, one has the following theorem. 
Theorem 9 ([15]) Let $T>0$. Let $X, Y$ and $R$ be three time-varying vector fields of period $T$ with respect to time. We assume that

$$
\begin{gathered}
X, Y, \text { and } R \text { are Carathéodory vector fields, } \\
Y \text { is } r \text {-homogeneous, } \\
X=Y+R .
\end{gathered}
$$

We also assume that, for some $\rho>0, \eta>0$ and $M>0$, one has

$$
\left|R_{i}\left(\varepsilon^{r_{1}} x_{1}, \ldots, \varepsilon^{r_{n}} x_{n}, t\right)\right| \leq M \varepsilon^{r_{i}+\eta}, \forall \varepsilon \in(0,1),
$$

for every $i \in\{1, \ldots, n\}$ and for every $x=\left(x_{1}, \ldots, x_{n}\right)^{t r} \in \mathbb{R}^{n}$ such that $|x| \leq \rho$. Let us assume that 0 is locally (or globally) asymptotically stable for $\dot{x}=Y(t, x)$. Then 0 is locally asymptotically stable for $\dot{x}=X(t, x)$ and there exists $\lambda>0, C>0$ and $\rho>0$ such that, for every solution $x:[0,+\infty) \rightarrow \mathbb{R}^{n}$ of $\dot{x}=X(t, x)$ such that

$$
\sum_{i=1}^{n}\left|x_{i}(0)\right|^{1 / r_{i}} \leqslant \rho
$$

one has

$$
\sum_{i=1}^{n}\left|x_{i}(t)\right|^{1 / r_{i}} \leqslant C e^{-\lambda t} \sum_{i=1}^{n}\left|x_{i}(0)\right|^{1 / r_{i}}, \quad \forall t \in[0,+\infty) .
$$

(In fact, in [15], Theorem 9 is stated with more regularity on the vector fields. However the Carathéodory regularity is in fact sufficient. See also (the proof of) Theorem 12.16 in [6], which relies on [16].)

Theorem 4 follows directly from Theorem 3 and Theorem 9 .

\section{Applications}

In this section we present various applications of our approach to construct stabilizing time-varying feedback laws.

\subsection{An example with $k=1$}

Let us consider the control system

$$
\dot{x}_{1}=x_{2}, \dot{x}_{2}=w, \dot{y}=x_{1}^{2}-x_{2}^{2},
$$

where the state is $\left(x_{1}, x_{2}, y\right)^{\operatorname{tr}} \in \mathbb{R}^{3}$ and the control is $w \in \mathbb{R}$. Let us recall the Poincare inequality

$$
\int_{0}^{1} \varphi(t)^{2} d t \leqslant \frac{1}{\pi^{2}} \int_{0}^{1} \dot{\varphi}(t)^{2} d t, \forall \varphi \in C^{1}([0,1]) \quad \text { such that } \quad \varphi(0)=\varphi(1)=0 .
$$

This inequality can be proved by expanding $\varphi$ as the following Fourier series

$$
\varphi(x)=\sum_{l=1}^{+\infty} f_{l} \sin (l \pi x)
$$


Indeed, with this expansion, one has for every $\varphi \in C^{1}([0,1])$ such that $\varphi(0)=\varphi(1)=0$,

$$
\int_{0}^{1} \varphi(t)^{2} d t=\sum_{l=1}^{+\infty} \frac{1}{2} f_{l}^{2} \leqslant \frac{1}{\pi^{2}} \sum_{l=1}^{+\infty} \frac{\pi^{2} l^{2}}{2} f_{l}^{2}=\frac{1}{\pi^{2}} \int_{0}^{1} \dot{\varphi}(t)^{2} d t .
$$

Note that $1 / \pi^{2}$ is optimal in $(4.2)$ as one can see by considering $\varphi(x):=\sin (\pi x)$. From inequality (4.2), one gets that the control system (4.1) is not (locally or globally) controllable in time $T$ if $T \leqslant \pi$. Moreover, using the fact that $1 / \pi^{2}$ is optimal in $(4.2)$ and using the power series expansion (see Section 1), one gets that the control system (4.1) is (locally and globally) controllable in time $T$ if $T>\pi$. However it does not satisfy the following necessary condition for feedback stabilization by means of continuous stationary feedback laws due to Brockett [1] (see also [6, Theorem 1.1]).

Theorem 10 Let us consider the control system $\dot{z}=f(z, w)$, where $z \in \mathbb{R}^{l}$ is the state, $w \in \mathbb{R}^{m}$ is the control. Assume that $f(0,0)=0$ and 0 can be locally asymptotically stabilized by means of continuous stationary feedback laws, i.e. there exists a continuous map $w: \mathbb{R}^{l} \rightarrow \mathbb{R}^{m}$ vanishing at 0 , such that 0 is (locally) asymptotically stable for the closed-loop system $\dot{z}=f(z, w(z))$. Then

the image by $f$ of every neighborhood of $(0,0) \in \mathbb{R}^{l} \times \mathbb{R}^{m}$ is a neighborhood of $0 \in \mathbb{R}^{l}$.

The control system (4.1) does not satisfy the Brockett condition (4.3). Indeed, if $(\alpha, \beta)^{\operatorname{tr}} \in \mathbb{R}^{2}$ is such that $\beta<-\alpha^{2}$, there is not $\left(x_{1}, x_{2}, w\right)^{\text {tr }} \in \mathbb{R}^{3}$ such that

$$
x_{2}=\alpha, w=0, x_{1}^{2}-x_{2}^{2}=\beta .
$$

Hence, by Theorem 10, the control system (4.1) cannot be locally asymptotically stabilized by means of continuous stationary feedback laws. We are going to see that Theorem 3 can be applied to construct time-varying stabilizing feedback laws. Let us first point out that 1.8$]$ is not satisfied. In order to deal with this problem it suffices to apply the transformation $w:=-x_{1}-x_{2}+u$, which transforms the control system (4.1) into the control system

$$
\dot{x}_{1}=x_{2}, \dot{x}_{2}=-x_{1}-x_{2}+u, \dot{y}=x_{1}^{2}-x_{2}^{2},
$$

where the state is $\left(x_{1}, x_{2}, y\right)^{\operatorname{tr}} \in \mathbb{R}^{3}$ and the control is $u \in \mathbb{R}$. This control system is of the form (1.1), with

$$
\begin{gathered}
A:=\left(\begin{array}{cc}
0 & 1 \\
-1 & -1
\end{array}\right), \quad B:=\left(\begin{array}{l}
0 \\
1
\end{array}\right), L:=0, \\
Q_{1}\left(x, x^{\prime}\right):=x_{1} x_{1}^{\prime}-x_{2} x_{2}^{\prime}, \quad \forall\left(x_{1}, x_{2}\right)^{\operatorname{tr}} \in \mathbb{R}^{2}, \forall\left(x_{1}^{\prime}, x_{2}^{\prime}\right)^{\operatorname{tr}} \in \mathbb{R}^{2}, Q_{2}:=0, Q_{3}:=0 .
\end{gathered}
$$

In order to apply Theorem 3 , let us first check that properties $\left(\mathcal{P}_{1}\right),\left(\mathcal{P}_{2}\right)$ and $\left(\mathcal{P}_{3}\right)$ are satisfied. Let $x=\left(x_{1}, x_{2}\right)^{\operatorname{tr}}:[0, T] \rightarrow \mathbb{R}^{2}$ be a solution of $\dot{x}=A x$. Then

$$
\begin{gathered}
\frac{d}{d t}\left(x_{1}^{2}+x_{2}^{2}\right)=-x_{2}^{2}, \\
\frac{d}{d t} x_{2}=-x_{1}-x_{2} \neq 0 \text { for }\left(x_{1}, x_{2}\right) \in(\mathbb{R} \backslash\{0\}) \times\{0\},
\end{gathered}
$$

which, together with the (proof of the) LaSalle invariance principle implies that $\left(\mathcal{P}_{1}\right)$ holds. Since $L=0$, one has $\left(\mathcal{P}_{2}\right)$. Let us now turn to $\left(\mathcal{P}_{3}\right)$, Let us first point that, by the Poincaré inequality (4.2), $\left(\mathcal{P}_{3}\right)$ cannot hold if $T \leqslant \pi$. Let us also mention that, by the controllability of 4.1) in time $T$ 
if $T>\pi$ and by Proposition 2 , $\left(\mathcal{P}_{3}\right)$ holds if $T>\pi$. Let us give an explicit $v$ having the properties required in $\left(\mathcal{P}_{3}\right)$. We take $T=3.6$ and define $v:[0, T] \times\{-1,1\} \rightarrow \mathbb{R}$ by

$$
v(t, b):= \begin{cases}g(t), & \text { if } b=-1 \text { and } 1 \leq t \leq T \\ 0, & \text { if } b=-1 \text { and } \quad \text { otherwise, } \\ f(t), & \text { if } b=1 \text { and } \quad T / 4 \leq t \leq(T+4) / 4\end{cases}
$$

with

$$
\begin{gathered}
f(t)=\frac{1}{10}\left(\left(t^{2}(12+t(4+t))-2 t(6+t(3+t)) T+(2+t(2+t)) T^{2}\right)\right) \\
g(t)=\frac{T^{4}}{2560}((2+(-1+t) t(10+t(3+t)))-128(-5+t(7+t(3+2 t))) T \\
\left.+16(7+6 t(1+t)) T^{2}-8(1+2 t) T^{3}+T^{4}\right) .
\end{gathered}
$$

These controls are represented on Fig. 1. Clearly $(1.10)$ and 1.11 hold.
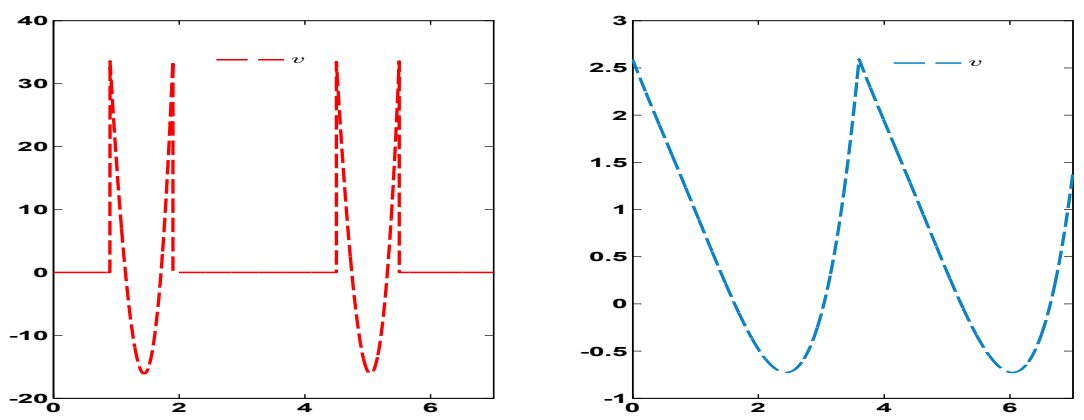

Figure 1: Control $v$ defined by 4.5 for $b=-1$ on the left and $b=1$ on the right.

Straightforward computations show that 1.12 holds, since, for $\tilde{y}$ defined in 1.12 , one has, for $b=1, \tilde{y}(3.6) * b=-4.92$ and, for $b=-1, \tilde{y}(3.6) * b=-0.12$. See also Fig. 2 .
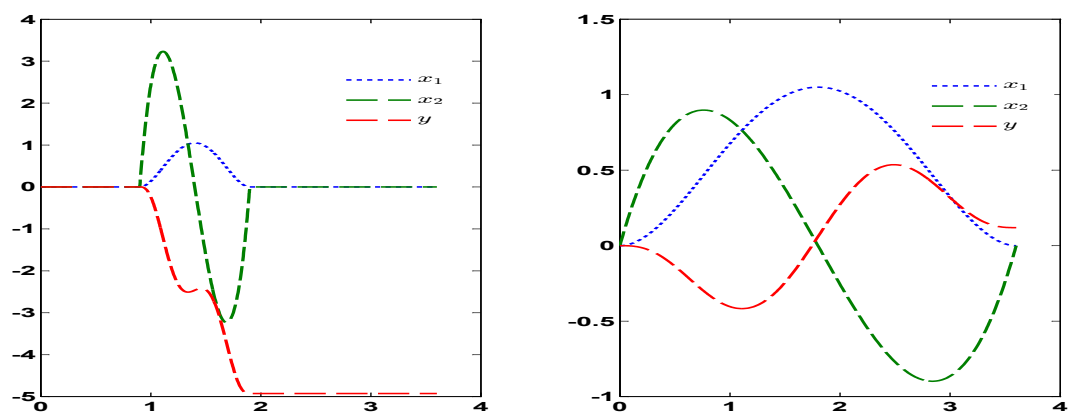

Figure 2: Solutions of 4.4 with $x(0)=0 \in \mathbb{R}^{2}, y(0)=0 \in \mathbb{R}$ and $u=v(t, b)$ for $v$ defined in 4.5), with $b=1$ on the left and $b=-1$ on the right. 
The feedback law $u$ defined by 1.13 and $(1.14)$ is

$$
u_{\varepsilon}(t, y)=\varepsilon \sqrt{|y|} v(t-4\lfloor t / 4\rfloor, \operatorname{Sign}(y))
$$

where, for $s \in \mathbb{R},\lfloor s\rfloor$ is the integer part of $s$ and $\operatorname{Sign}(s)$ is the $\operatorname{sign}$ of $s: \operatorname{Sign}(s)=1$ for $s \in(0,+\infty)$, $\operatorname{Sign}(s)=-1$ for $s \in(-\infty, 0)$ and $\operatorname{Sign}(0)=0$.
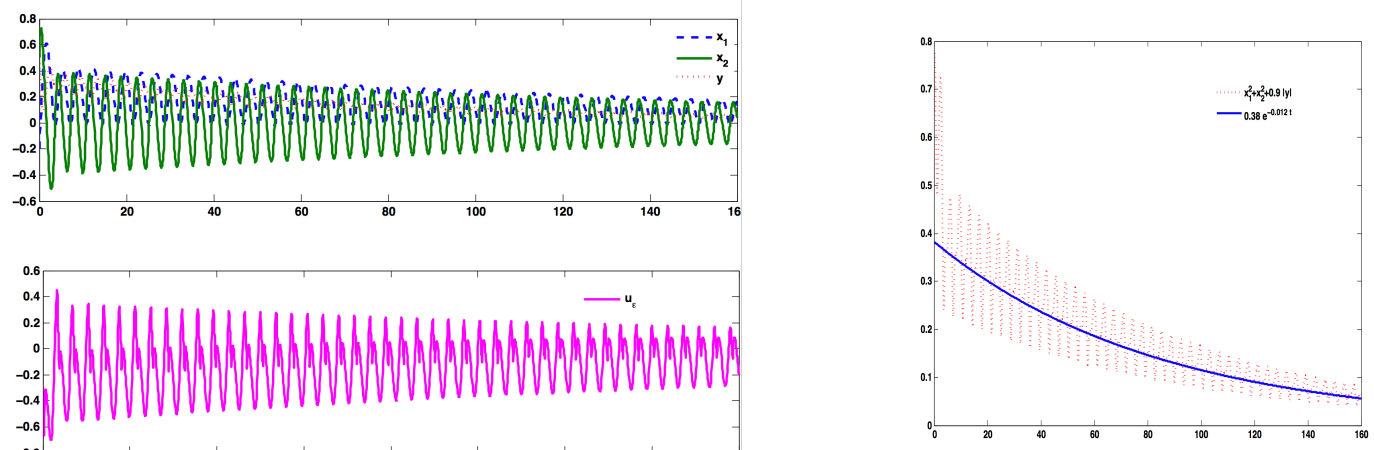

Figure 3: Trajectory of the solution of the control system 4.1). For $\varepsilon=0.9$ and initial data $x_{1}(0)=$ $-0.2, x_{2}(0)=0.5$ and $y(0)=0.46$ and comparison of decay between $\left|\left(x_{1}, x_{2}\right)^{\operatorname{tr}}\right|^{2}+0.9|y|$ and $y(t)=$ $0.38 \exp (-0.012 t)$.
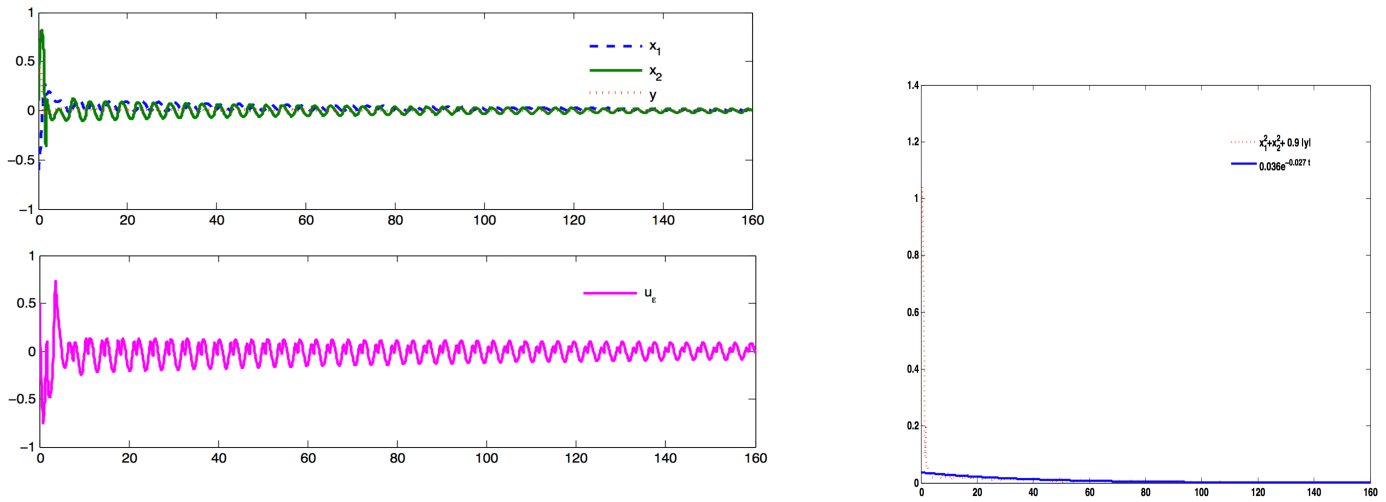

Figure 4: Trajectory of the solution of the control system 4.1). For $\varepsilon=0.9$ and initial data $x_{1}(0)=-0.6$, $x_{2}(0)=0.1$ and $y(0)=-0.2$ and comparison of decay between $\left|\left(x_{1}, x_{2}\right)^{\operatorname{tr}}\right|^{2}+0.9|y|$ and $0.037 \exp (-0.027 t)$.

\subsection{An example with $k=2$}

We consider the control system

$$
\dot{x}_{1}=w_{1}, \quad \dot{x}_{2}=w_{2}, \quad \dot{y}_{1}=x_{1}^{2}-x_{2}^{2}, \quad \dot{y}_{2}=2 x_{1} x_{2},
$$

where the state is $z:=\left(x_{1}, x_{2}, y_{1}, y_{2}\right)^{\operatorname{tr}} \in \mathbb{R}^{4}$ and the control is $u=\left(w_{1}, w_{2}\right)^{\operatorname{tr}} \in \mathbb{R}^{2}$. One easily check that, for every $T>0$, this control system is (globally) controllable in time $T$. This control 
system satisfies the Brockett condition 4.3. However one has the following necessary condition, due to Coron [2] for feedback stabilization by means of stationary feedback laws, a condition which is slightly stronger than the Brockett condition (4.3).

Theorem 11 If the control system $\dot{z}=f(z, v)$, where $z \in \mathbb{R}^{l}$ is the state, $v \in \mathbb{R}^{m}$ is the control and $f(0,0)=0$, can be locally asymptotically stabilized by means of continuous stationary feedback laws, then, for $\varepsilon>0$ small enough,

$$
\left.f_{*}\left(H_{l-1}\left(\left\{(z, u) \in \mathbb{R}^{n} ; f(z, u) \neq 0,|z|<\varepsilon \text { and }|u|<\varepsilon\right\}\right)\right)\right)=H_{l-1}\left(\mathbb{R}^{l} \backslash\{0\}, \mathbb{Z}\right),
$$

where $H_{l-1}(\Omega, \mathbb{Z})$ denotes the $(l-1)$-singular homology group of $\Omega$ with integer coefficients and $f_{*}$ is the homomorphism induced by $f:\left\{(z, u) \in \mathbb{R}^{n} ; f(z, u) \neq 0,|z|<\varepsilon\right.$ and $\left.|u|<\varepsilon\right\} \rightarrow \mathbb{R}^{l} \backslash\{0\}$ (see, e.g., [20, page 161]).

The control system (4.6) does not satisfy the Coron condition in Theorem 11. Indeed, the control system (4.6) can be written as $\dot{z}=f(z, u)$, with for $z=\left(x_{1}, x_{2}, y_{1}, y_{2}\right)^{\operatorname{tr}} \in \mathbb{R}^{4}$ and $u=\left(w_{1}, w_{2}\right)^{\operatorname{tr}} \in$ $\mathbb{R}^{2}$,

$$
f(z, u)=\left(w_{1}, w_{2}, x_{1}^{2}-x_{2}^{2}, 2 x_{1} x_{2}\right)^{\operatorname{tr}} .
$$

Then one can check that, for every $\varepsilon>0$,

$$
\left.f_{*}\left(H_{3}\left(\left\{(z, u) \in \mathbb{R}^{4} \times \mathbb{R}^{2} ; f(z, u) \neq 0,|z|<\varepsilon \text { and }|u|<\varepsilon\right\}\right), \mathbb{Z}\right)\right)=2 H_{3}\left(\mathbb{R}^{4} \backslash\{0\}, \mathbb{Z}\right) .
$$

By Theorem 11, since the control system (4.6) does not satisfy (11), it cannot be asymptotically stabilized by means of (continuous) stationary feedback laws. However it is locally controllable in small-time and, by [5], it can be asymptotically stabilized by means of periodic time-varying feedback laws. Let us check that, once more, the method presented in this article allows to construct such feedback laws.

As in the previous application in Section 4.1, condition $\left(\mathcal{P}_{1}\right)$ is not satisfied. In order to deal with this problem it suffices to apply the transformation $w_{1}=-x_{1}+u_{1}$ and $w_{2}=-x_{2}+u_{2}$, which transforms the control system 4.6 into

$$
\dot{x}_{1}=-x_{1}+u_{1}, \quad \dot{x}_{2}=-x_{2}+u_{2}, \dot{y}_{1}=x_{1}^{2}-x_{2}^{2}, \quad \dot{y}_{2}=2 x_{1} x_{2},
$$

where the state is $x=\left(x_{1}, x_{2}, y_{1}, y_{2}\right)^{\operatorname{tr}} \in \mathbb{R}^{4}$ and the control is $u=\left(u_{1}, u_{2}\right)^{\operatorname{tr}} \in \mathbb{R}^{2}$. This control system has the form (1.1), with $n=m=k=2$,

$$
\begin{gathered}
A:=\left(\begin{array}{cc}
-1 & 0 \\
0 & -1
\end{array}\right), B:=\left(\begin{array}{cc}
1 & 0 \\
0 & 1
\end{array}\right), L:=0 \\
Q_{1}\left(x, x^{\prime}\right):=\left(x_{1} x_{1}^{\prime}-x_{2} x_{2}^{\prime}, 2 x_{1} x_{2}^{\prime}\right)^{\operatorname{tr}}, \forall\left(x_{1}, x_{2}\right)^{\operatorname{tr}} \in \mathbb{R}^{2}, \forall\left(x_{1}^{\prime}, x_{2}^{\prime}\right)^{\operatorname{tr}} \in \mathbb{R}^{2}, Q_{2}:=0, Q_{3}:=0 .
\end{gathered}
$$

Then $\left(\mathcal{P}_{1}\right)$ is satisfied with $\rho_{1}:=e^{-T} \in(0,1)$. Clearly 4.8 implies that $\left(\mathcal{P}_{2}\right)$ holds. By the controllability of (4.6) in time $T$ for every $T>0$ and by Proposition $2,\left(\mathcal{P}_{3}\right)$ holds for every $T>0$. Let us give an explicit $v$ having the properties required in $\left(\mathcal{P}_{3}\right)$. We choose $T:=4$ and consider the control law $v:[0, T] \times \mathbb{S}^{1} \rightarrow \mathbb{R}^{2}$ defined by

$$
\begin{aligned}
& v_{1}(t, b)=-\frac{3 \sqrt{70}}{512} \operatorname{Sign}\left(b_{2}\right) \sin \left(\left(\arccos \left(b_{1}\right)\right) / 2\right) t(t-4)\left(t^{2}-8\right), \\
& v_{2}(t, b)=-\frac{3 \sqrt{70}}{512} \operatorname{Sign}\left(b_{2}\right) \cos \left(\left(\arccos \left(b_{1}\right)\right) / 2\right) t(t-4)\left(t^{2}-8\right),
\end{aligned}
$$


with $v=\left(v_{1}, v_{2}\right)^{\operatorname{tr}}$ and $b=\left(b_{1}, b_{2}\right)^{\operatorname{tr}}$. This control is represented on Fig. 5 and 6 .
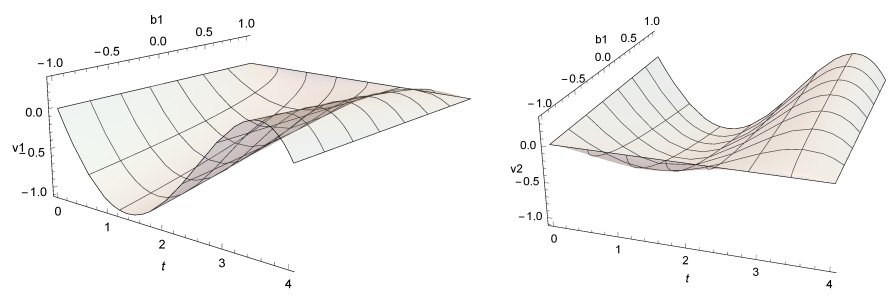

Figure 5: Controls $v_{1}(t, b)$ and $v_{2}(t, b)$ defined in 4.10 and 4.11 for $t \in[0,4]$ and $b \in \mathbb{S}^{1}$ respectively when $b_{2}$ is negative.
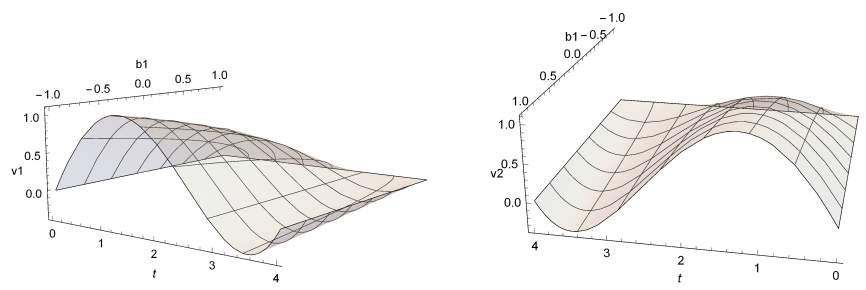

Figure 6: Controls $v_{1}(t, b)$ and $v_{2}(t, b)$ defined in (4.10) and 4.11) for $t \in[0,4]$ and $b \in \mathbb{S}^{1}$ respectively when $b_{2}$ is positive.

In figure 7 the condition condition $\left(\mathcal{P}_{3}\right)$ can be verified.
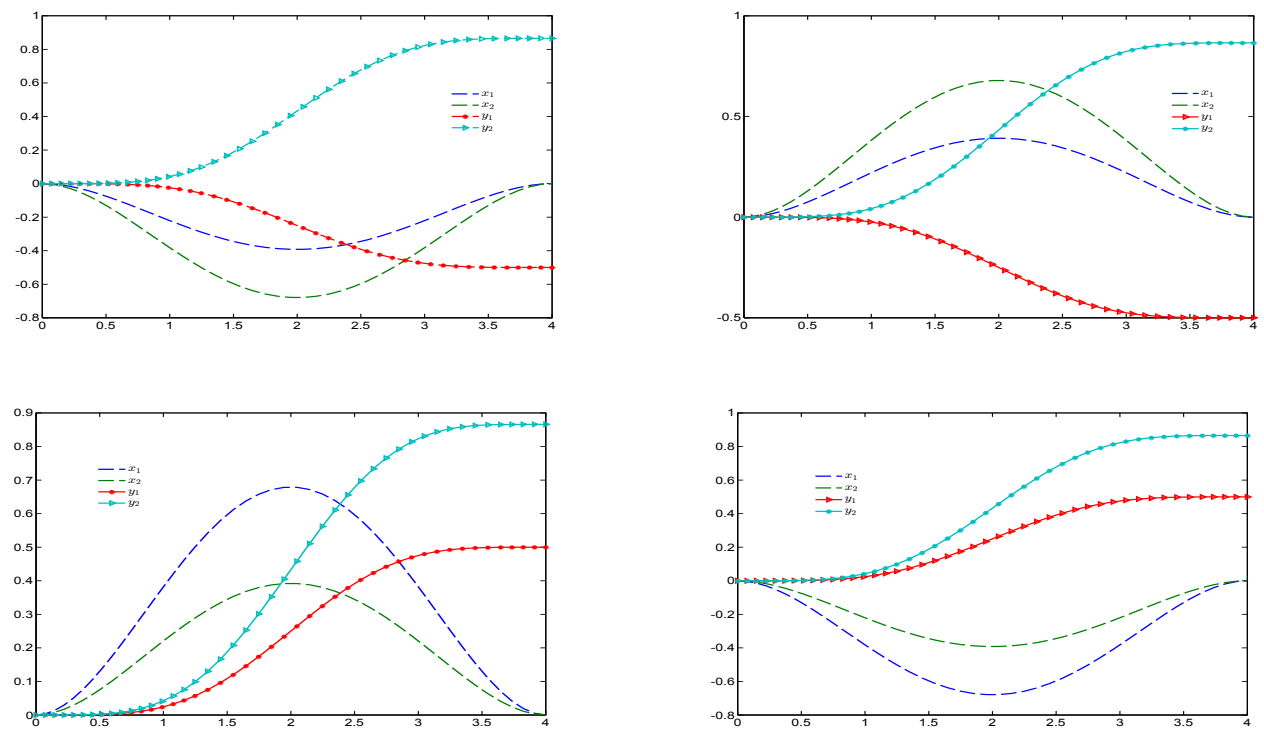

Figure 7: Solutions of 4.7) with $x(0)=0 \in \mathbb{R}^{2}, y(0)=0 \in \mathbb{R}^{2}$ and $v=v(t, b)=\left(v_{1}(t, b), v_{2}(t, b)\right)$ where $v$ is defined in (4.10) and (4.11) when $b \in \mathbb{S}^{1}$ takes the values $(0.5,0.86)^{\operatorname{tr}},(-0.5,0.86)^{\operatorname{tr}},(-0.5,-0.86)^{\operatorname{tr}}$ and $(0.5,0.86)^{\text {tr }}$ respectively. 
For the control system (4.7), we consider the feedback law

$$
u_{i}(t, y)=u_{i \varepsilon}(t, y)=\varepsilon \sqrt{|y|} v_{i}(t, y /|y|), i=1,2 .
$$

In Figure 8 and 9, a trajectory of system 4.7) with the feedback law 4.12 is shown.
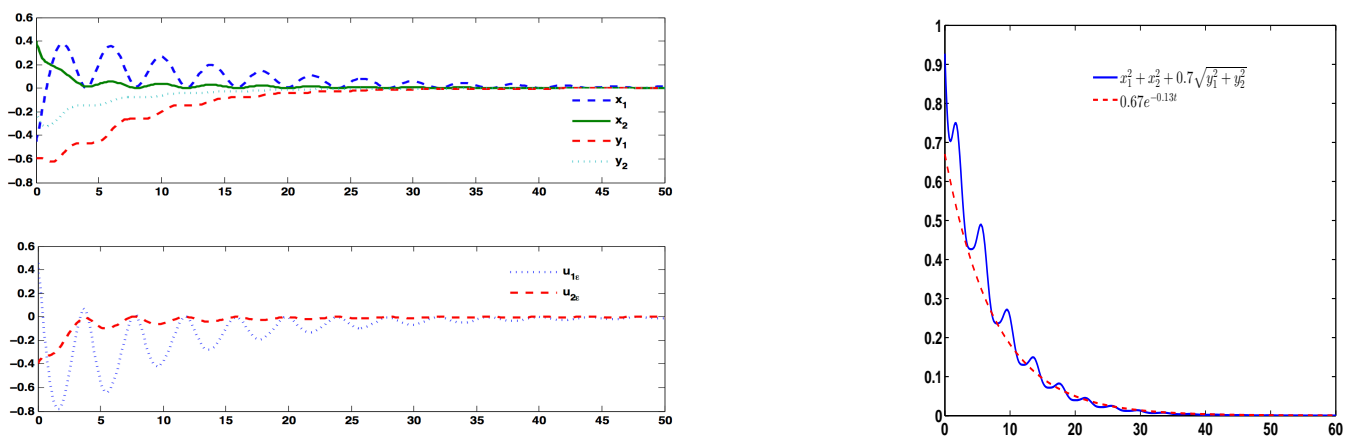

Figure 8: Trajectory of the solution of the control system (4.7) for $\varepsilon=0.7$ and initial data $x_{1}(0)=-0.4$, $x_{2}(0)=0.4, y_{1}(0)=-0.6$ and $y_{2}(0)=-0.2$, and comparison of the decay rate between $\left|\left(x_{1}, x_{2}\right)^{\operatorname{tr}}\right|^{2}+$ $\varepsilon\left|\left(y_{1}, y_{2}\right)^{\mathrm{tr}}\right|$ and $0.67 \exp (-0.13 t)$.
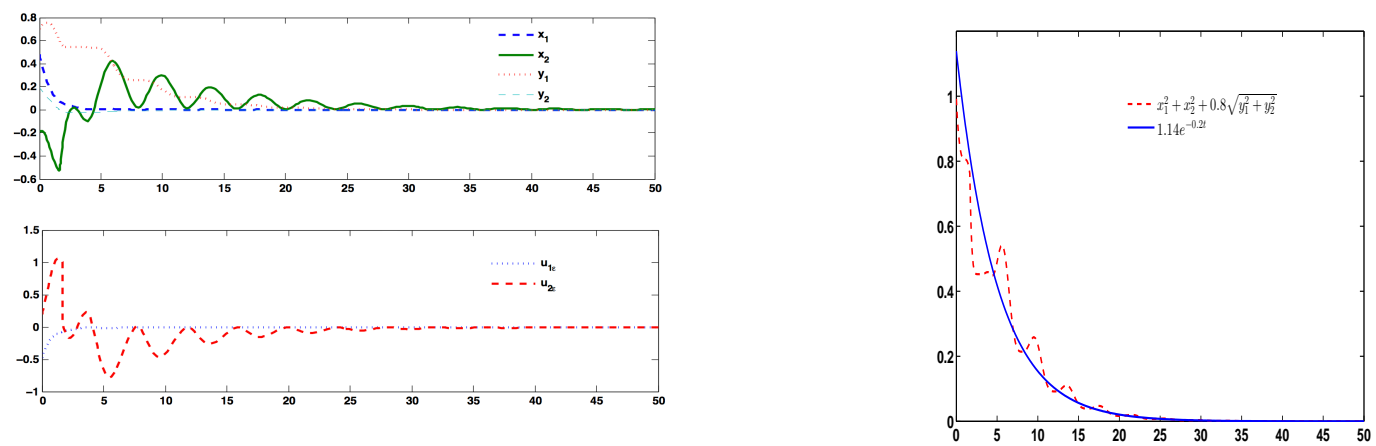

Figure 9: Trajectory of the solution of the control system 4.7) for $\varepsilon=0.8$ and initial data $x_{1}(0)=-0.48$, $x_{2}(0)=-0.2, y_{1}(0)=0.7$ and $y_{2}(0)=0.2$ and comparison of the decay rate between $\left|\left(x_{1}, x_{2}\right)^{\operatorname{tr}}\right|^{2}+\varepsilon\left|\left(y_{1}, y_{2}\right)^{\operatorname{tr}}\right|$ and $1.14 \exp (-0.2 t)$. 


\subsection{The baby stroller control system}

In this section, we consider the following nonlinear system, describing the motion of a baby stroller (or unicycle),

$$
\dot{x}_{1}=w_{1} \cos x_{2}, \dot{x}_{2}=w_{2}, \dot{y}=w_{1} \sin x_{2},
$$

where $x_{1}$ and $y$ are the coordinates of the midpoint between the two back wheels, $x_{2}$ is an angle which gives the orientation of the baby stroller (see Figure 10 . For this control system, the state is $\left(x_{1}, x_{2}, y\right)^{\operatorname{tr}} \in \mathbb{R}^{3}$ and the control is $\left(w_{1}, w_{2}\right)^{\operatorname{tr}} \in \mathbb{R}^{2}$.

By a theorem due to Rashevski and Chow (see, for example, [6, Theorem 3.19]), the control system (4.13) is locally controllable in time $T$ for every $T>0$. The control system 4.13) does not satisfy the Brockett condition (4.3), as it can be easily checked by looking at the solution of

$$
w_{1} \cos x_{2}=0, w_{2}=0, w_{1} \sin x_{2}=\varepsilon
$$

where the unknown is $\left(x_{1}, x_{2}, y, w_{1}, w_{2}\right)^{\text {tr }} \in \mathbb{R}^{5}$ and the data is $\varepsilon \neq 0$. Hence, by Theorem 10 , the control system (4.13) cannot be locally asymptotically stabilized by means of continuous stationary feedback laws. Let us emphasize that, however, by [3], we know that the control system 4.13) can be asymptotically stabilized by means of periodic time-varying feedback laws. Various explicit stabilizing periodic time-varying feedback laws for the control system 4.13) were constructed. Let us just mention Samson's pioneer work [17, as well as [7]. Let us show how Theorem 4 can be applied in order to construct such stabilizing feedback laws.

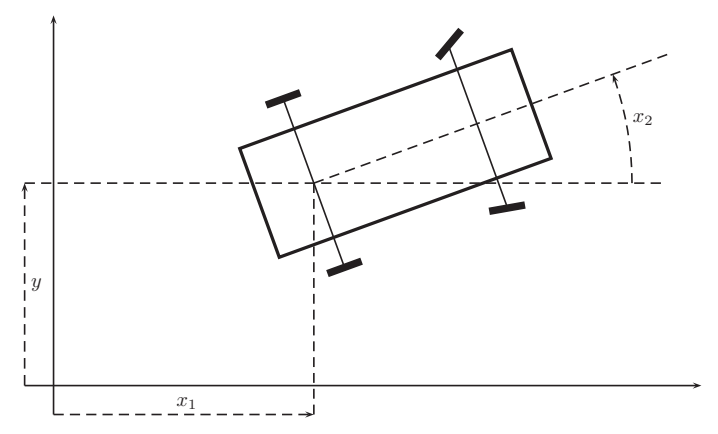

Figure 10: Baby Stroller design of system 4.13

The quadratic approximation (in the sense explained in the introduction) of 4.13 around $(\overrightarrow{0}, 0) \in$ $\mathbb{R}^{3} \times \mathbb{R}$ is

$$
\dot{x}_{1}=w_{1}, \dot{x}_{2}=w_{2}, \dot{y}=w_{1} x_{2},
$$

where the state is $\left(x_{1}, x_{2}, y\right)^{\operatorname{tr}} \in \mathbb{R}^{3}$ and the control is $\left(w_{1}, w_{2}\right)^{\operatorname{tr}} \in \mathbb{R}^{2}$. 
The control system (4.14) has the form of (1.1). However it does not satisfies Property ( $\left.\mathcal{P}_{1}\right)$, In order to handle this problem, we perform the following change of variables

$$
u_{1}=w_{1}+x_{1}, u_{2}=w_{2}+x_{2},
$$

which transforms the control system 4.14 into the control system

$$
\dot{x}_{1}=-x_{1}+u_{1}, \dot{x}_{2}=-x_{2}+u_{2}, \dot{y}=-x_{1} x_{2}+x_{2} u_{1},
$$

where the state is $\left(x_{1}, x_{2}, y\right)^{\operatorname{tr}} \in \mathbb{R}^{3}$ and the control is $\left(u_{1}, u_{2}\right)^{\operatorname{tr}} \in \mathbb{R}^{2}$. It still has the form (1.1) with $n=2, m=2, k=1$,

$$
\begin{gathered}
A:=\left(\begin{array}{cc}
-1 & 0 \\
0 & -1
\end{array}\right), B:=\left(\begin{array}{ll}
1 & 0 \\
0 & 1
\end{array}\right), \\
Q_{1}(x, \tilde{x}):=-\frac{1}{2}\left(x_{1} \tilde{x}_{2}+\tilde{x}_{1} x_{2}\right), Q_{2}(x, u)=x_{2} u_{1}, Q_{3}(u, \tilde{u})=0,
\end{gathered}
$$

for every $x=\left(x_{1}, x_{2}\right)^{\operatorname{tr}} \in \mathbb{R}^{2}$, every $\tilde{x}=\left(\tilde{x}_{1}, \tilde{x}_{2}\right)^{\operatorname{tr}} \in \mathbb{R}^{2}$, every $u=\left(u_{1}, u_{2}\right)^{\operatorname{tr}} \in \mathbb{R}^{2}$ and every $\tilde{u} \in \mathbb{R}^{2}$. Property $\left(\mathcal{P}_{1}\right)$ now holds. For the control system $(4.15)$, one has $L=0$ and therefore Property $\left(\mathcal{P}_{2}\right)$ holds. Let us now consider Property $\left(\mathcal{P}_{3}\right)$. By a theorem due to Sussmann [22], the control system (4.15) is locally controllable in time $T$ for every $T>0$ and, therefore, by Proposition 2 , $\left(\mathcal{P}_{3}\right)$ holds for every $T>0$. Let us give an explicit $u$ having the properties required in $\left(\mathcal{P}_{3}\right)$. We take $T:=3$. Let $a_{ \pm}=\left(2187-280 \delta \mp 140 \varepsilon_{1}\right) / 2187$ with $\delta>0$ as in $\left(\mathcal{P}_{3}\right)$ and $\varepsilon_{1}>0$. Let us define $v=\left(v_{1}, v_{2}\right)^{\operatorname{tr}}:[0,3] \times\{-1,1\} \rightarrow \mathbb{R}^{2}$ by

$$
\begin{gathered}
v_{1}(t, \pm 1)=a_{ \pm}\left(t^{4}-6 t^{3}+10 t^{2}-t-3\right), \forall t \in[0,3], \\
v_{2}(t, 1)=-v_{2}(t,-1)=t^{4}-2 t^{3}+3 t^{2}-18 t+18, \forall t \in[0,3] .
\end{gathered}
$$

The controls $t \in[0, T] \mapsto v(t, b)$ with $b \in \mathbb{S}^{0}=\{-1,1\}$ are plotted on Figure 11
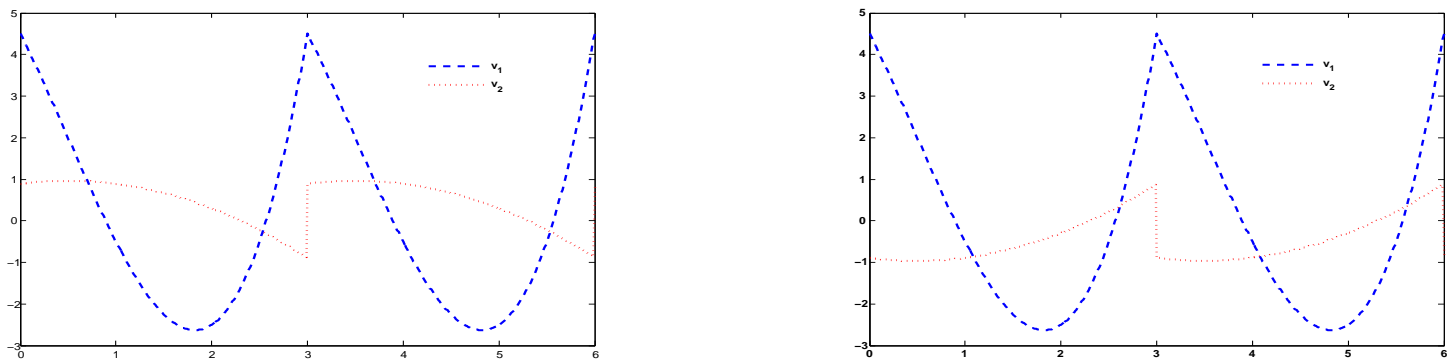

Figure 11: Controls (4.16) and 4.17) for $b=-1$ on the left and for $b=1$ on the right.

Clearly (1.10) and 1.11) hold. Straightforward computations give that, if we take $2 \delta=0.5$ and $\varepsilon_{1}=0.12$, then, for $\tilde{y}$ defined in $(1.12)$, for $b=-1, \tilde{y}(3)=1.49$ and for $b=1, \tilde{y}(3)=-1.49$, which 
shows that 1.12 holds.
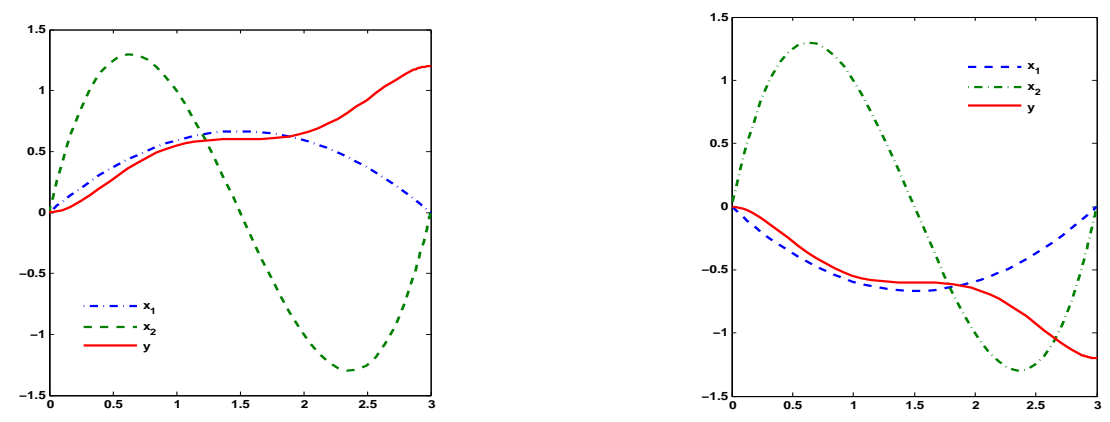

Figure 12: Solutions of 4.15 with $x(0)=0 \in \mathbb{R}^{2}, y(0)=0 \in \mathbb{R}$ and $u=v(t, b)$ for $v$ defined in 4.16 and (4.17), with $b=-1$ on the and $b=1$ on the right.

For $\varepsilon>0$, we define $u_{\varepsilon}=\left(u_{1 \varepsilon}, u_{2 \varepsilon}\right)^{\text {tr }}: \mathbb{R} \times \mathbb{R} \rightarrow \mathbb{R}^{2}$ by 1.13 and (1.14). Let

$$
\rho_{2} \in\left(\rho_{1}, 1\right) \text {. }
$$

We may now apply Proposition 6 . Let $\varepsilon_{0}>0$ and $C>0$ be as in this proposition and let $\varepsilon \in\left(0, \varepsilon_{0}\right]$. By Proposition 6, we get that

$$
\begin{gathered}
|x(t)|^{2}+\varepsilon|y(t)| \leqslant C\left(|x(0)|^{2}+\varepsilon|y(0)|\right), \forall t \in[0, T], \\
|x(T)|^{2}+\varepsilon|y(T)| \leqslant \rho_{2}|x(0)|^{2}+\varepsilon\left(1-\varepsilon^{2} \delta\right)|y(0)|
\end{gathered}
$$

for every solution $(x, y):[0, T] \rightarrow \mathbb{R}^{2} \times \mathbb{R}$ of the closed-loop system

$$
\dot{x}_{1}=-x_{1}+u_{\varepsilon 1}, \dot{x}_{2}=-x_{2}+u_{\varepsilon 2}(t, y), \dot{y}=-x_{1} x_{2}+x_{2} u_{\varepsilon 1}(t, y) .
$$

Let us now check, using Theorem 4, that the same time-varying feedback also leads to asymptotic stability for the initial control system $(4.13)$, i.e. that $0 \in \mathbb{R}^{3}$ is also (locally) asymptotically stable for

$$
\dot{x}_{1}=\left(-x_{1}+u_{\varepsilon 1}(t, y)\right) \cos x_{2}, \dot{x}_{2}=-x_{2}+u_{\varepsilon 2}(t, y), \dot{y}=\left(-x_{1}+u_{\varepsilon 1}(t, y)\right) \sin x_{2} .
$$

Let $R_{x}: \mathbb{R}^{2} \times \mathbb{R} \times \mathbb{R}^{2} \rightarrow \mathbb{R}^{2}$ and $R_{y}: \mathbb{R}^{2} \times \mathbb{R} \times \mathbb{R} \rightarrow \mathbb{R}$ be defined by

$$
R_{x}(x, y, u):=\left(\left(-x_{1}+u_{1}\right)\left(-1+\cos x_{2}\right), 0\right)^{\operatorname{tr}}, R_{y}(x, y, u):=\left(-x_{1}+u_{1}\right)\left(-x_{2}+\sin x_{2}\right),
$$

for every $x=\left(x_{1}, x_{2}\right)^{\operatorname{tr}} \in \mathbb{R}^{2}$, every $y \in \mathbb{R}$ and every $u=\left(u_{1}, u_{2}\right)^{\operatorname{tr}} \in \mathbb{R}^{2}$. Then 4.13$)$ is the control system 1.19). Let us point out that there exists $M_{1}>0$ such that

$$
\left|x_{3}\left(-1+\cos x_{2}\right)\right| \leqslant M_{1}\left(\left|x_{2}\right|+\left|x_{3}\right|\right)^{3},\left|x_{3}\left(-x_{2}+\sin x_{2}\right)\right| \leqslant M_{1}\left(\left|x_{2}\right|+\left|x_{3}\right|\right)^{4}, \forall\left(x_{2}, x_{3}\right)^{\operatorname{tr}} \in \mathbb{R}^{2} .
$$

In particular 1.20 and 1.21 hold for $\eta:=2>0$ provided that $M>0$ is large enough. Hence, using Theorem 4 , one gets the following proposition. 
Proposition 12 There exists $\varepsilon_{0}>0$ such that, for every $\varepsilon \in\left(0, \varepsilon_{0}\right]$, there exist $C>0, \rho>0$ and $\lambda>0$ such that, for every solution $(x, y)$ of 4.18 with $|x(0)|^{2}+|y(0)| \leqslant \rho$, one has

$$
|x(t)|^{2}+|y(t)| \leqslant C e^{-\lambda t}\left(|x(0)|^{2}+|y(0)|\right), \forall t \in[0,+\infty) .
$$

Numerical simulations are presented on Fig. 13 and Fig. 14.
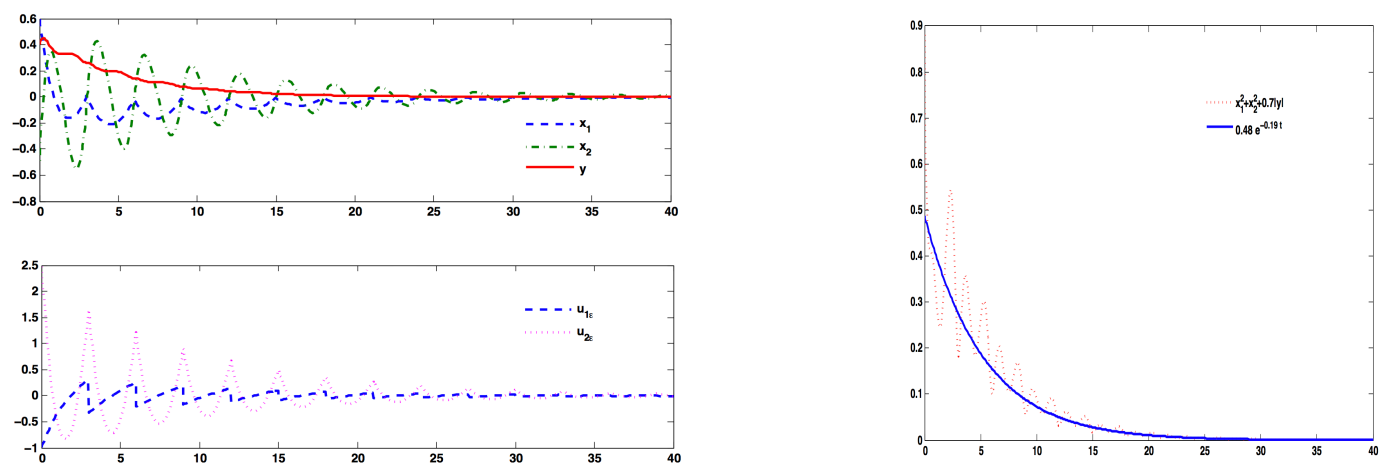

Figure 13: Trajectory of the closed-loop control system $(4.13)$ for $\varepsilon=0.7$ and initial data $x_{1}(0)=0.6$, $x_{2}(0)=0.4$, and $y(0)=-0.49$ and comparison of the decay rate between $\left|\left(x_{1}, x_{2}\right)^{\operatorname{tr}}\right|^{2}+0.7|y|$ and $0.48 \exp (-0.19 t)$.
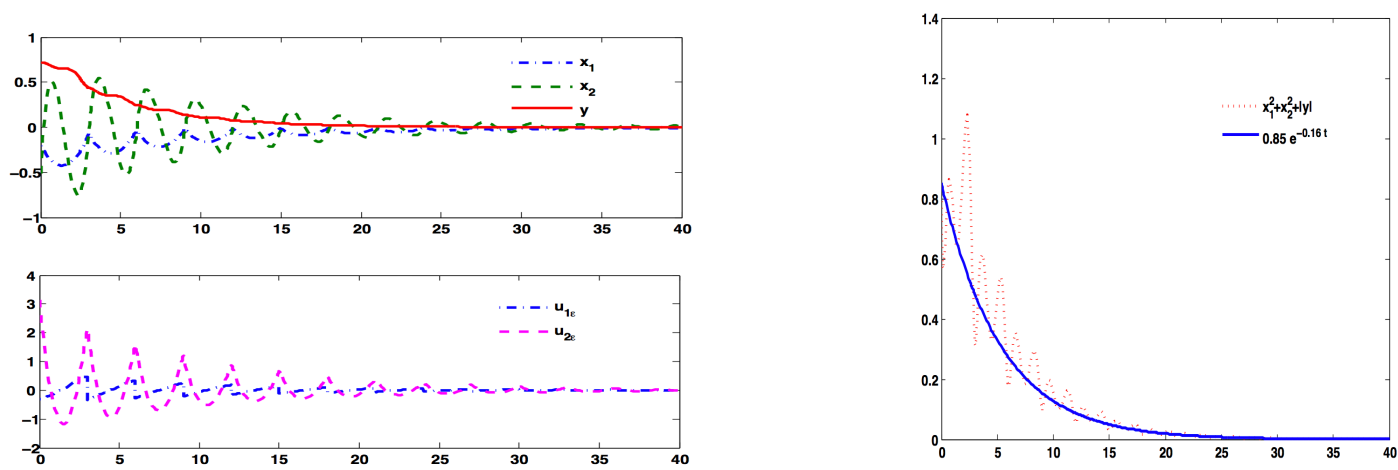

Figure 14: Trajectory of the closed-loop control system 4.13 for $\varepsilon=0.7$ and initial data $x_{1}(0)=-0.2$, $x_{2}(0)=0.7$, and $y(0)=-0.5$ and comparison of the decay rate between $\left|\left(x_{1}, x_{2}\right)^{\operatorname{tr}}\right|^{2}+0.7|y|$ and $0.85 \exp (-0.19 t)$.

\subsection{Underactuated surface vessel system}

Let us consider the nonlinear system that describes the dynamic positioning of surface vessels which describes drilling, pipe-laying and diving support. More precisely, a ship that has not side thruster, 
but two independent main thrusters located at a distance from the center line in order to provide both surge force and yaw moment.

$$
\begin{gathered}
\dot{u}=-\frac{d_{1}}{m_{1}} u+\frac{m_{2}}{m_{1}} v r+\frac{1}{m_{1}} \tau_{1}, \quad \dot{v}=-\frac{m_{1}}{m_{2}} u r-\frac{d_{2}}{m_{2}} v, \quad \dot{r}=-\frac{d_{3}}{m_{3}} r+\frac{m_{1}-m_{2}}{m_{3}} u v+\frac{1}{m_{3}} \tau_{3}, \\
\dot{z}_{1}=u+z_{2} r, \quad \dot{z}_{2}=v-z_{1} r, \quad \dot{z}_{3}=r,
\end{gathered}
$$

where the state is $\left(u, v, r, z_{1}, z_{2}, z_{3}\right)^{\operatorname{tr}} \in \mathbb{R}^{6}$ and the control is $\left(\tau_{1}, \tau_{3}\right) \in \mathbb{R}^{2}$. Physically, $u, v$ and $r$ are the velocities in surge, sway and yaw respectively, the parameters $m_{i}>0$ and $d_{i}>0$ for $i=1,2,3$ are the ship inertia, added mass effect and hydrodynamic damping, $\tau_{1}$ is the surge control force and $\tau_{3}$ is the yaw control moment. Once more,

- using [22], one gets that the control system 4.19)-4.20 is locally controllable in small time,

- the control system 4.19-4.20 does not satisfy the Brockett condition 4.3 and, therefore, by Theorem 10, the control system (4.19)- 4.20 cannot be locally asymptotically stabilized by means of continuous stationary feedback laws,

- by [5, the control system 4.19-4.20 can be asymptotically stabilized by means of periodic time-varying feedback laws.

Let us point out that time-varying stabilizing feedback laws for the control system (4.19)-(4.20) have been constructed by Mazenc, Pettersen and Nijmeijer in [13. Let us show how Theorem 3 can be used in order to construct other time-varying stabilizing feedback laws.

Before treating the system, we perform transformations already presented in [13. We do the following change of variables

$$
\begin{gathered}
Z_{2}=z_{2}+\frac{m_{2}}{d_{2}} v, u=-\frac{d_{2}}{m_{1}}\left(z_{1}-\mu\right) \\
\tau_{r}=\frac{m_{1}-m_{2}}{m_{3}} u v-\frac{d_{3}}{m_{3}} r+\frac{1}{m_{3}} \tau_{3}, \\
\tau_{\mu}=\frac{d_{2}}{m_{2}} z_{1}+\frac{d_{2}}{m_{2}} \mu-Z_{2} r+\frac{m_{2}}{d_{2}} v r-\frac{1}{d_{2}}\left(m_{2} v r-d_{1} u+\tau_{1}\right)
\end{gathered}
$$

Then the control system (4.19)- 4.20) becomes the control system

$$
\begin{gathered}
\dot{z}_{1}=-\frac{d_{2}}{m_{1}} z_{1}-\frac{d_{2}}{m_{1}} \mu+Z_{2} r-\frac{m_{2}}{d_{2}} v r, \quad \dot{Z}_{2}=\mu r, \quad \dot{z}_{3}=r, \\
\dot{v}=-\frac{d_{2}}{m_{2}} v+\frac{d_{2}}{m_{2}}\left(z_{1}+\mu\right) r, \quad \dot{\mu}=\tau_{\mu}, \quad \dot{r}=\tau_{r},
\end{gathered}
$$

where the state is $\left(z_{1}, Z_{2}, z_{3}, v, \mu, r\right)^{\operatorname{tr}} \in \mathbb{R}^{5}$ and the control is $\left(\tau_{\mu}, \tau_{r}\right)^{\operatorname{tr}} \in \mathbb{R}^{2}$.

From now on, in order to simplify the notations, we consider the case where the physical constants are equal to 1 . i.e. $m_{i}=1$ and $d_{i}=1$ for $i=1,2$. Once more a preliminary change of variables is necessary to guaranty that the system satisfies the assumptions of Theorem 4 . We perform the following change of variables

$$
\tau_{\mu}=-\mu+\tau_{\mu}^{*} \quad \text { and } \quad \tau_{r}=-2 z_{3}-r+\tau_{r *} .
$$


With the notations of Section 1, $x:=\left(z_{1}, \mu, z_{3}, r\right)^{\operatorname{tr}} \in \mathbb{R}^{4}$ and $y:=\left(v, Z_{2}\right)^{\operatorname{tr}}$. Then the control system has the form (1.1) with $n=4, m=2, k=2$,

$$
\begin{gathered}
A:=\left(\begin{array}{cccc}
-1 & -1 & 0 & 0 \\
0 & -1 & 0 & 0 \\
0 & 0 & 0 & 1 \\
0 & 0 & -2 & -1
\end{array}\right), \quad B:=\left(\begin{array}{ll}
0 & 0 \\
1 & 0 \\
0 & 0 \\
0 & 1
\end{array}\right), \\
L=\left(\begin{array}{cc}
-1 & 0 \\
0 & 0
\end{array}\right), \quad Q_{1}(x, x)=\left(\begin{array}{c}
\left(z_{1}+\mu\right) r \\
\mu r
\end{array}\right), Q_{2}:=0, Q_{3}:=0 .
\end{gathered}
$$

Simple computations show

$$
\begin{aligned}
\left\|e^{A T}\right\|_{2} & =\left\|\left(\begin{array}{cccc}
\frac{1}{e^{4}} & -\frac{1}{e^{4}} & 0 & 0 \\
0 & \frac{1}{e^{4}} & 0 & 0 \\
0 & 0 & \frac{\sin (2 \sqrt{7})}{\sqrt{7} e^{2}}+\frac{\cos (2 \sqrt{7})}{e^{2}} & \frac{2 \sin (2 \sqrt{7})}{\sqrt{7} e^{2}} \\
0 & 0 & -\frac{4 \sin (2 \sqrt{7})}{\sqrt{7} e^{2}} & \frac{\cos (2 \sqrt{7})}{e^{2}}-\frac{\sin (2 \sqrt{7})}{\sqrt{7} e^{2}}
\end{array}\right)\right\|_{2} \\
& =\max \left\{\frac{1}{e^{4}},\left|\frac{7 e^{2} \cos (2 \sqrt{7})-7 i e^{2} \sin (2 \sqrt{7})}{7 e^{4}}\right|,\left|\frac{7 e^{2} \cos (2 \sqrt{7})+7 i e^{2} \sin (2 \sqrt{7})}{7 e^{4}}\right|\right\} \\
& =0.13,
\end{aligned}
$$

hence, the property $\left(\mathcal{P}_{1}\right)$ holds for $T=4$. Property $\left(\mathcal{P}_{2}\right)$ follows from 4.25$)$. Again, by using 22 , one gets that the control system (1.1), with $A, B, L$ and $Q$ defined by (4.24) and (4.25), is locally controllable in time $T$ for every $T>0$ and by Proposition $2,\left[\left(\mathcal{P}_{3}\right)\right.$ holds for every $T>0$. Let us, once more, give an explicit $v$ having the properties required in $\left[\left(\mathcal{P}_{3}\right)\right.$. We again choose $T=4$ and define the "control $v$ " for Property $\left[\mathcal{P}_{3}\right)$ by

$$
\tau_{\mu}^{*}:=\frac{\left(-2338875 b_{2}+4096 b_{1} e^{4}+41895 b_{2} e^{4}\right)\left(32+16 t-20 t^{2}+t^{4}\right)}{32768 c\left(-30499+559 e^{4}\right)}
$$

and

$$
\begin{array}{r}
\tau_{r}^{*}:=2 c \frac{-256 b_{1} e^{4}\left(-256+416 t-304 t^{2}+156 t^{3}-49 t^{4}+6 t^{5}\right)}{4096 b_{1} e^{4}+315 b_{2}\left(-7425+133 e^{4}\right)}+ \\
\frac{315 b_{2}\left(-118800+10056 t-49578 t^{2}+34270 t^{3}-1771 t^{4}-1028 t^{5}\right)}{4096 b_{1} e^{4}+315 b_{2}\left(-7425+133 e^{4}\right)}+ \\
\frac{315 b_{2}\left(e^{4}\left(2128-104 t+850 t^{2}-598 t^{3}+23 t^{4}+20 t^{5}\right)\right)}{4096 b_{1} e^{4}+315 b_{2}\left(-7425+133 e^{4}\right)}
\end{array}
$$

for $c=\frac{1}{4}$, see figure 15 

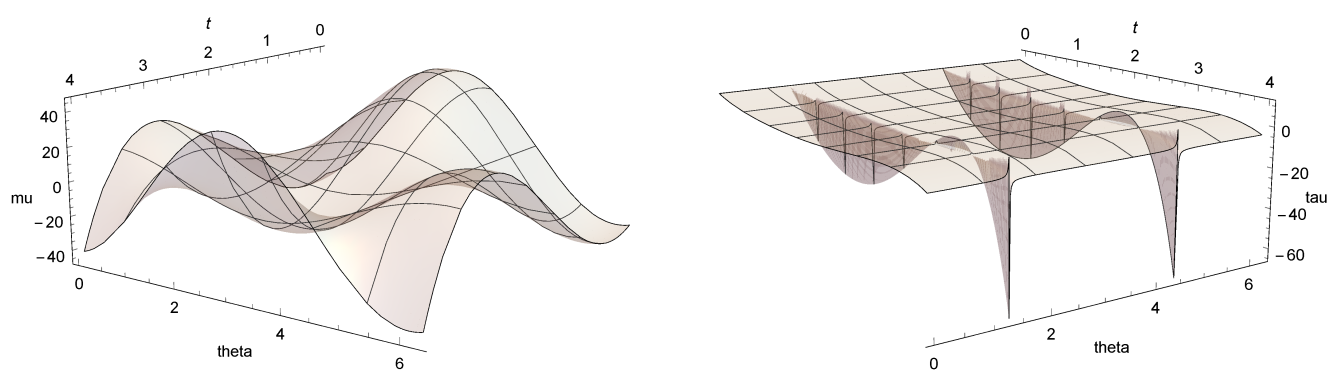

Figure 15: Controls $\tau_{\mu}$ and $\tau_{r}$ defined in 4.23) with 4.26) and 4.27 respectively, with $b_{1}=\cos \theta$ and $b_{2}=\sin \theta$ for $\theta \in[0,2 \pi]$.
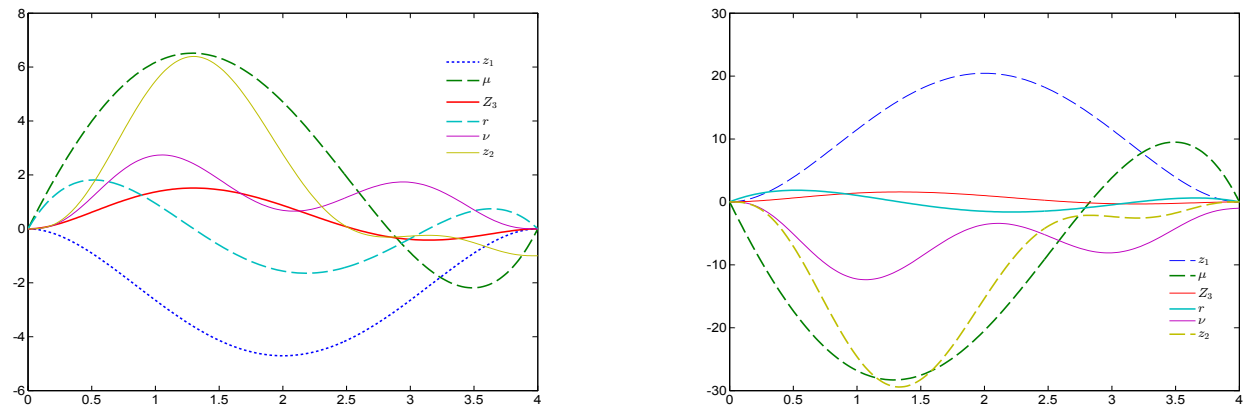

Figure 16: Solution of 4.21)-4.22)-(4.23) starting from $0 \in \mathbb{R}^{6}$, with $v=\left(\tau_{\mu}, \tau_{r}\right)$ with $\tau_{\mu}^{*}$ defined in 4.26), $\tau_{r}^{*}$ defined in 4.27), for $b=(0,1)^{\operatorname{tr}}$ on the left and for $b=(1,0)^{\operatorname{tr}}$ on the right.

Trajectories of the closed-loop system are shown on Figures 17 and 18 for $\varepsilon=0.5$ and for different values of the initial data.
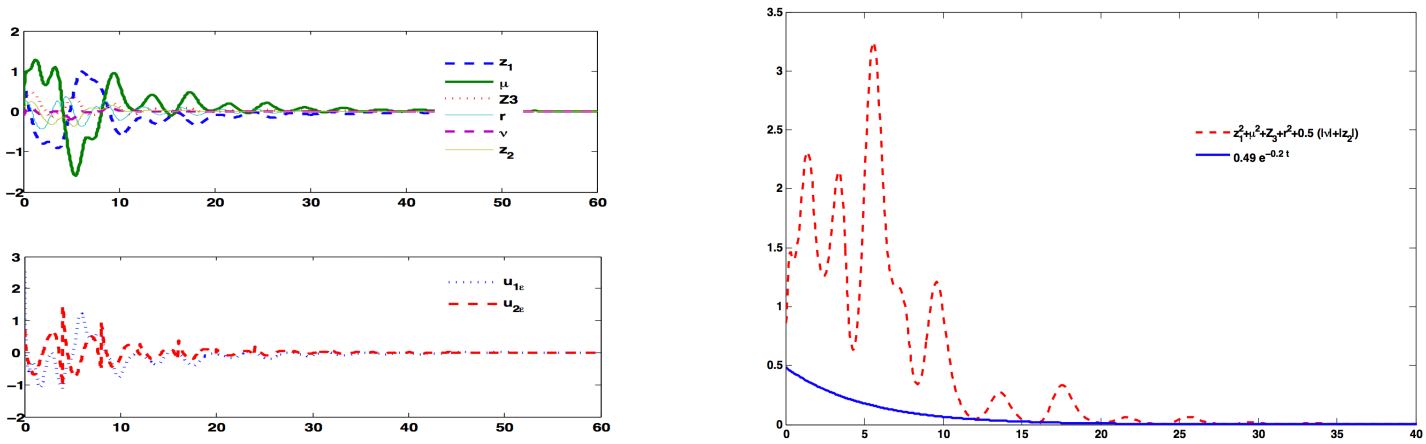

Figure 17: Trajectory of the solution of the control system 4.21) and 4.22 for $\varepsilon=0.5$ and the initial data $z_{1}(0)=0.8, z_{3}(0)=0.3, \mu(0)=0.2, r(0)=0.1, v(0)=-0.2, z_{2}(0)=0.5$. and comparison of the decay rate between $\left|\left(z_{1}, z_{3}, \mu, r\right)^{\operatorname{tr}}\right|^{2}+0.5\left|\left(v, z_{2}\right)^{\operatorname{tr}}\right|$ and $0.49 \exp (-0.2 t)$. 

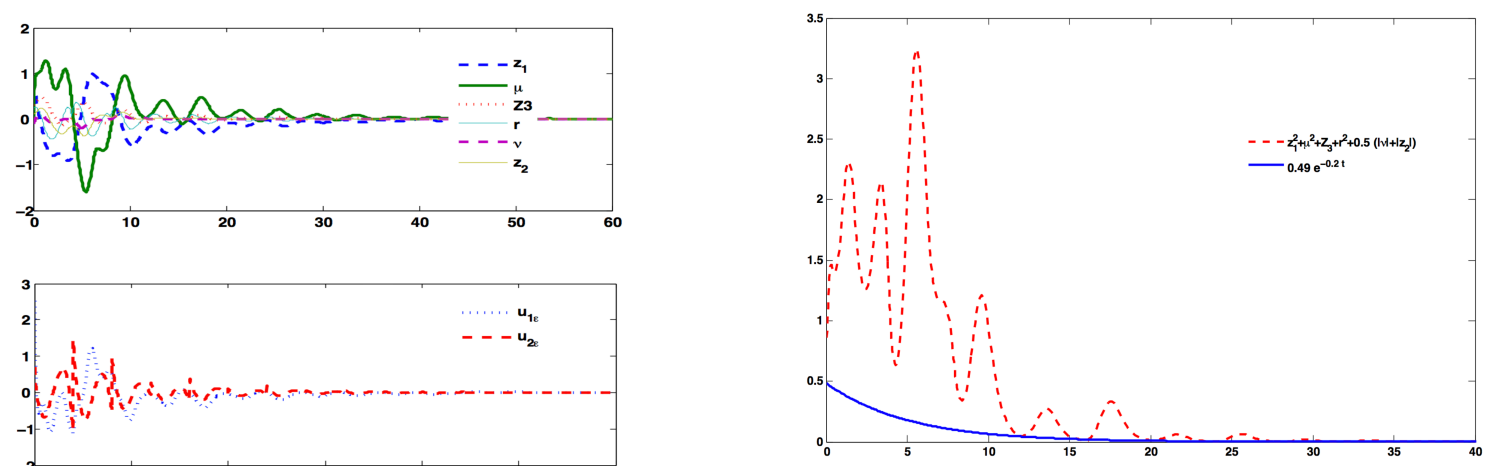

Figure 18: Trajectory of the solution of the control system 4.21) and 4.22 for $\varepsilon=0.5$ and the initial data $z_{1}(0)=0.56, z_{3}(0)=-0.3, \mu(0)=0.2, r(0)=0.1, v(0)=-0.2$ and $z_{2}(0)=0.5$ and comparison of decay rate between $\left|\left(z_{1}, z_{3}, \mu, r\right)^{\operatorname{tr}}\right|^{2}+0.5\left|\left(v, z_{2}\right)^{\operatorname{tr}}\right|$ and $7.38 \exp (-0.15 t)$.

\section{Open Problems}

In the literature, there are interesting models that do not adapt to our study, as the rigid spacecraft [9] described by the control system

$$
\begin{gathered}
\dot{x}_{1}=x_{5} x_{6}, \dot{x}_{2}=x_{1}+c x_{3} x_{6}, \dot{x}_{3}=x_{5}, \\
\dot{x}_{5}=u_{1}, \dot{x}_{6}=u_{2}
\end{gathered}
$$

where the state is $\left(x_{1}, x_{2}, x_{3}, x_{5}, x_{6}\right)^{\operatorname{tr}} \in \mathbb{R}^{5}$ and the control $\left(u_{1}, u_{2}\right)^{\operatorname{tr}} \in \mathbb{R}^{2}$, and $c \neq 0$, as well the X4-flyer modeled by (see [23])

$$
\begin{gathered}
\dot{x}=x_{1}, \dot{x}_{1}=\frac{1}{m} \alpha \sin (\beta), \dot{y}=y_{1}, \dot{y}_{1}=\frac{1}{m} \alpha \cos (\beta), \dot{\alpha}=u, \\
\dot{\beta}=\varphi, \dot{z}=z_{1}, \dot{z}_{1}=g-\frac{1}{m} \nu, \dot{\varphi}=\omega, \dot{\omega}=\tau_{\varphi}
\end{gathered}
$$

where the state is $\left(x, x_{1}, y, y_{1}, z, z_{1}, \alpha, \beta, \varphi, \omega\right)^{\operatorname{tr}} \in \mathbb{R}^{10}$ and the control is $\left(u, \nu, \tau_{\varphi}\right)^{\operatorname{tr}} \in \mathbb{R}^{3}$.

For the satellite,

$$
L:=\left(\begin{array}{ll}
0 & 0 \\
1 & 0
\end{array}\right)
$$

and, for the X4-flyer,

$$
L:=\left(\begin{array}{cc}
0 & -1 \\
0 & 0
\end{array}\right) .
$$

Therefore, these two problems do not satisfy Property $\left(\mathcal{P}_{2}\right)$.

\section{A Proof of Proposition 2}

Proof of Proposition 2. Let us assume, for the moment, that the following lemma holds. 
Lemma 13 Let $\tau \in(0, T)$. Let us assume that the control system (1.1) is locally controllable in time $\tau$. Then there exists $\bar{u} \in L^{\infty}\left([0, T] ; \mathbb{R}^{m}\right)$ such that, if $(\bar{x}, \bar{y}) \in C^{0}\left([0, T] ; \mathbb{R}^{n} \times \mathbb{R}^{k}\right)$ is the solution of

$$
\dot{\bar{x}}=A \bar{x}+B \bar{u}(t), \quad \dot{\bar{y}}=L \bar{y}+Q_{1}(\bar{x}, \bar{x})+Q_{2}(\bar{x}, \bar{u})+Q_{3}(\bar{u}, \bar{u}), \quad \bar{x}(0)=0, \quad \bar{y}(0)=0,
$$

then the following two properties hold

$$
\bar{x}(T)=0 \text { and } \bar{y}(T)=0,
$$

the linearized control system control system of (1.1) around $\left(\left(\bar{x}^{t r}, \bar{y}^{t r}\right)^{t r}, \bar{u}\right)$ is controllable.

For $r>0$, let $B_{r}^{k}$ be the open ball of radius $r$ centered at $0 \in \mathbb{R}^{k}$. Using the inverse mapping theorem and Lemma 13 , there exists $r>0$ and $W \in C^{1}\left(B_{r}^{k} ; L^{\infty}\left([0, T] ; \mathbb{R}^{m}\right)\right)$ such that, for every $b \in B_{r}^{k}$,

$$
\begin{aligned}
& \left(\dot{x}=A x+B W(b)(t), \dot{y}=L y+Q_{1}(x, x)+Q_{2}(x, W(b)(t))\right. \\
& \left.+Q_{3}(W(b)(t), W(b)(t)), x(0)=0, y(0)=0\right) \Rightarrow(x(T)=0, y(T)=b) .
\end{aligned}
$$

Let us now define $v:[0, T] \times \mathbb{S}^{k-1} \rightarrow \mathbb{R}^{m}$ by

$$
v(t, b):=W\left(-e^{T L} \frac{r b}{2\left\|e^{T L}\right\|_{2}}\right)(t), \quad \forall(t, b) \in[0, T] \times \mathbb{S}^{k-1} .
$$

Then one has (1.10), and if $C_{0}>0$ is large enough, (1.11) holds. Moreover, from (A.4) and (A.5), one has, for every $b \in \mathbb{S}^{k-1}$,

$$
\begin{aligned}
(\dot{\tilde{x}}=A \tilde{x}+ & B v(t, b), \dot{\tilde{y}}=L \tilde{y}+Q_{1}(\tilde{x}, \tilde{x})+Q_{2}(x, v(t, b)) \\
& \left.+Q_{3}(v(t, b), v(t, b)), \tilde{x}(0)=0, \tilde{y}(0)=0\right) \Rightarrow\left(\tilde{x}(T)=0, \tilde{y}(T)=-e^{T L} \frac{r b}{2\left\|e^{T L}\right\|_{2}}\right)
\end{aligned}
$$

which shows that 1.12 holds with

$$
\delta:=\frac{r}{4\left\|e^{T L}\right\|_{2}\left\|e^{-T L}\right\|_{2}^{2}} .
$$

Finally, let us prove Lemma 13. By the R. Hermann [11] and T. Nagano [14 theorem and the controllability assumption, the control system (1) satisfies the Lie algebra rank condition at $(0,0) \in$ $\mathbb{R}^{n+k} \times \mathbb{R}^{m}$ (see, e.g., [6, Definition 3.16]). Then, by [4, Theorem 1.3], for every $\eta>0$, there exists $\bar{u} \in C^{\infty}\left([0, T-\tau] ; \mathbb{R}^{m}\right)$ such that, if $(\bar{x}, \bar{y}) \in C^{\infty}\left([0, T-\tau] ; \mathbb{R}^{n} \times \mathbb{R}^{k}\right)$ is the solution of A.1) on $[0, T-\tau]$, then the following two properties hold

$$
|\bar{x}(T-\tau)|+|\bar{y}(T-\tau)|<\eta,
$$

the linearized control system control system of (1.1) around $\left(\left(\bar{x}^{\operatorname{tr}}, \bar{y}^{\operatorname{tr}}\right)^{\operatorname{tr}}, \bar{u}\right)$ is controllable. (A.9)

We choose $\eta>0$ as in Definition 1. Then, from (A.8) we can extend $\bar{u}$ to $[0, T]$ so it is an element of $L^{\infty}\left([0, T] ; \mathbb{R}^{m}\right)$ such that, if $(\bar{x}, \bar{y})$ is extended to $[0, T]$ so it is the element of $C^{0}\left([0, T] ; \mathbb{R}^{n} \times \mathbb{R}^{k}\right)$ which is the solution of A.1 on $[0, T]$, then $A .2$ holds. Then A.9 implies A.3. This concludes the proof of Lemma 13 and, also, the proof of Proposition 2.

Remark 14 An alternative proof of Proposition 2 can be provided by using [18] instead of [4]. 


\section{References}

[1] Roger W. Brockett. Asymptotic stability and feedback stabilization. In Differential geometric control theory (Houghton, Mich., 1982), volume 27 of Progr. Math., pages 181-191. Birkhäuser Boston, Boston, MA, 1983.

[2] Jean-Michel Coron. A necessary condition for feedback stabilization. Systems Control Lett., 14(3):227-232, 1990.

[3] Jean-Michel Coron. Global asymptotic stabilization for controllable systems without drift. Math. Control Signals Systems, 5(3):295-312, 1992.

[4] Jean-Michel Coron. Linearized control systems and applications to smooth stabilization. SIAM J. Control Optim., 32(2):358-386, 1994.

[5] Jean-Michel Coron. On the stabilization in finite time of locally controllable systems by means of continuous time-varying feedback law. SIAM J. Control Optim., 33(3):804-833, 1995.

[6] Jean-Michel Coron. Control and nonlinearity, volume 136 of Mathematical Surveys and Monographs. American Mathematical Society, Providence, RI, 2007.

[7] Jean-Michel Coron. Stabilization of control systems and nonlinearities. In Proceedings of the 8th International Congress on Industrial and Applied Mathematics, pages 17-40. Higher Ed. Press, Beijing, 2015.

[8] Jean-Michel Coron and Emmanuelle Crépeau. Exact boundary controllability of a nonlinear KdV equation with critical lengths. J. Eur. Math. Soc. (JEMS), 6(3):367-398, 2004.

[9] Jean-Michel Coron and El-Yazid Keraï. Explicit feedbacks stabilizing the attitude of a rigid spacecraft with two control torques. Automatica J. IFAC, 32(5):669-677, 1996.

[10] Jean-Michel Coron, Ivonne Rivas, and Shengquan Xiang. Local exponential stabilization for a class of Korteweg-de Vries equations with time-varying feedback law. Prepint, 2016.

[11] Robert Hermann. On the accessibility problem in control theory. In Internat. Sympos. Nonlinear Differential Equations and Nonlinear Mechanics, pages 325-332. Academic Press, New York, 1963.

[12] Jaroslav Kurzweil. On the inversion of Lyapunov's second theorem on stability of motion. Ann. Math. Soc. Trans. Ser.2,, 24:19-77, 1956.

[13] Frédéric Mazenc, Kristin Pettersen, and Henk Nijmeijer. Global uniform asymptotic stabilization of an underactuated surface vessel. IEEE Trans. Automat. Control, 47(10):1759-1762, 2002 .

[14] Tadashi Nagano. Linear differential systems with singularities and an application to transitive Lie algebras. J. Math. Soc. Japan, 18:398-404, 1966.

[15] Jean-Baptiste Pomet and Claude Samson. Exponential stabilization of nonholonomic systems in power form. In IFAC Symposium on Robust Control Design (Rio de Janeiro 1994), pages 447-452. Pergamon, Oxford, 1994. 
[16] Lionel Rosier. Homogeneous Lyapunov function for homogeneous continuous vector fields. Systems Control Lett., 19(6):467-473, 1992.

[17] Claude Samson. Velocity and torque feedback control of a nonholonomic cart. In C. Canudas de Wit, editor, Advanced robot control (Grenoble, 1990), volume 162 of Lecture Notes in Control and Inform. Sci., pages 125-151. Springer, Berlin, 1991.

[18] Eduardo D. Sontag. Finite-dimensional open-loop control generators for nonlinear systems. Internat. J. Control, 47(2):537-556, 1988.

[19] Eduardo D. Sontag and Héctor J. Sussmann. Remarks on continuous feedback. In Proc. IEEE Conf. Decision and Control, Albuquerque (1980), pages 916-921. IEEE, New York, 1980.

[20] Edwin H. Spanier. Algebraic topology. McGraw-Hill Book Co., New York-Toronto, Ont.London, 1966.

[21] Héctor J. Sussmann. Subanalytic sets and feedback control. J. Differential Equations, 31(1):3152, 1979.

[22] Héctor J. Sussmann. A general theorem on local controllability. SIAM J. Control Optim., 25(1):158-194, 1987.

[23] Naim Zoghlami, Lotfi Beji, Rhouma Mlayeh, and Azgal Abichou. The finite-time stability of perturbed systems. IEEE, pages 1080-1085, 2012. 